\title{
A Quantum Version of Spectral Decomposition Theorem of Dynamical Systems, Quantum Chaos Hierarchy: Ergodic, Mixing and Exact

\author{
Ignacio Gomez ${ }^{1}$ and Mario Castagnino ${ }^{2}$
}

September 3, 2018

1- Instituto de Física de Rosario (IFIR-CONICET), Rosario, Argentina
2- Instituto de Física de Rosario (IFIR-CONICET) and
Instituto de Astronomía y Física del Espacio (IAFE-CONICET),
Casilla de Correos 67, Sucursal 28, 1428 Buenos Aires, Argentina.

\begin{abstract}
In this paper we study Spectral Decomposition Theorem 1 and translate it to quantum language by means of the Wigner transform. We obtain a quantum version of Spectral Decomposition Theorem (QSDT) which enables us to achieve three distinct goals: First, to rank Quantum Ergodic Hierarchy levels [2, 3. Second, to analyze the classical limit in quantum ergodic systems and quantum mixing systems. And third, and maybe most important feature, to find a relevant and simple connection between the first three levels of quantum ergodic hierarchy (ergodic, exact and mixing) and quantum spectrum. Finally, we illustrate the physical relevance of QSDT applying it to two examples: Microwave billiards [4, 5] and a phenomenological Gamow model type [6, 7] .
\end{abstract}

Key words: QSDT-QEH-ergodic-mixing-exact

\section{Introduction}

Dynamical systems are one of most extensively studied subjects in physics. Mathematically, a dynamical system can be defined as a quadruplet $(X, \Sigma, \mu, \tau)$ where $X$ is a set (typically, the phase space in classical mechanics), $\Sigma$ is a sigma-algebra on $X, \mu$ is a finite measure on $\Sigma$ and map $\tau: X \rightarrow X$ is a measure-preserving transformation (see section 2.1). Physical interpretation of this abstract definition is that a dynamical system gives a fixed rule which describes time dependence of a point (state of system) in a geometrical space (the phase space). This rule is deterministic in the sense that for a given time interval only one future state follows from the current state.

From its origins in Newtonian mechanics to its subsequent measure theoretical definition numerous tools have been developed both theoretical and practical in dynamical systems theory. Some of these are discrete maps, bifurcation theory, topological knots, etc. These give different 


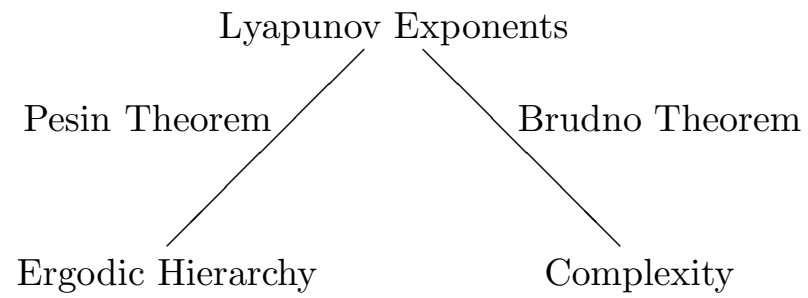

Figure 1: "Chaos pyramid" is a diagram for relationships between Ergodic Hierarchy, Lyapunov exponents and complexity through Pesin and Brudno theorems.

description 1 of dynamical systems. However, in many cases most important is the asymptotic behavior of dynamical system Z(mathematically, $t \rightarrow \infty$ ). This is the case of approach to equilibrium and we can use tools of chaos theory to study the evolution to equilibrium. Related to this, classical chaos presents several approaches which are related to each other: algorithmic complexity [8, Lyapunov exponents [9, 10] and Ergodic Hierarchy [1, 11. For instance, Brudno theorem 8 relates complexity with Kolmogorov-Sinai entropy [8 while Pesin theorem 8 relates Ergodic Hierarchy with Lyapunov exponents. The relationships between these chaos indicators is illustrated in the "chaos pyramid" of Fig. 1. According to this structure, Ergodic Hierarchy is one of features of classical chaos.

Ergodic Hierarchy ranks the chaotic level of a dynamical system according to the way in which correlations between two arbitrary distributions cancel for large times $(t \rightarrow \infty)$. For example, in classical mechanics a typical correlation $C(A, B)$, between two sets $\mathrm{A}$ and $\mathrm{B}$ of the phase space $\mathcal{M}$, is $C(A, B)=\mu(A \cap B)-\mu(A) \mu(B)$ where $\mu$ is a measure defined over subsets of $\mathcal{M}$. If $\mu(\mathcal{M})=1$ then $\mu(A)$ can be interpreted as probability of $A$. In such case, $C(A, B)$ can be interpreted as the difference between probability of $A$ and $B$ simultaneously and product of probabilities of $A$ and $B$. In this sense $C(A, B)$ is a correlation that "measures" how independent are events $A$ and $B$. Moreover, Ergodic Hierarchy ranks chaotic level of a dynamical system through correlations $C(A, B)$. For the quantum case, Quantum Ergodic Hierarchy (QEH) [2, 3 ] also expresses how correlations are canceled for large times, but in this case quantum correlations are defined between states and observables as quantum mean values $(\rho(t) \mid O)$.

On the other hand, we have an important tool called Spectral Decomposition Theorem (SDT) [1] which describes asymptotic behavior of dynamical systems. Roughly speaking and assuming certain hypotheses about the evolution of system, it says that any density of a dynamical system tends asymptotically $(t \rightarrow \infty)$ to a sum of localized densities (more precisely, normalized characteristic functions). As we mentioned above, Ergodic Hierarchy ranks asymptotically chaos between two distributions or densities. So we can expect some kind of connection between Ergodic Hierarchy and Spectral Decomposition Theorem. In fact, a connection is established in Theorem 8 (see section 3) which gives necessary and sufficient conditions for ergodic, mixing and exact levels of Ergodic Hierarchy according to SDT decomposition.

For quantum systems, quantum chaos is a discipline in constant evolution which began in 70's with pioneering experiments of microwave billiards [4, 12, 15, 16] and it is well known as the study of quantum mechanical aspects of quantum systems which have a chaotic classical description. Typical approaches that emerged from this evolution were scars [4, 12], WKB approximation [4, 17, and Random Matrix Theory [4, 15. A reasonable definition that takes into account the main characteristics of quantum chaos was given by M. Berry [18]: "a quantum

\footnotetext{
${ }^{1}$ More precisely, they give local and global descriptions of flux of a dynamical system. Since in this paper we will not use concept of flow, we will not expose any discussion about it. For more details see [9, 10, 12,

${ }^{2}$ For example, in synchronized dynamical systems like Kuramoto model (see p. 42 of [13], p. 164 of [14]).
} 
system is chaotic if its classical limit is chaotic". This was taken later on to be a possible definition of quantum chaos.

As we mention in introduction of part one [2, that there are many ways to define quantum chaos by complexity (see [19, 20]), exponential divergence trajectories (see [21, 22]), treatment of chaos based on the introduction of non-linear terms in the Schrodinger equation [23] and non-unitary evolution of a quantum system as an indicator of quantum chaos [24]. However, as mentioned in 2, the study of chaos based on QEH takes into account that classical limit may not exhibit chaotic behavior which would be a threat to Correspondence Principle (CP). In paper [25] we discuss and give an alternative way to study chaos in quantum systems based on fundamental graininess and the classical statistical limit which is compatible with CP.

As in previous works [2, 3, 25], in this paper we study quantum chaos from Berry's definition and Quantum Ergodic Hierarchy [2].

The main goal of this paper is a Quantum Version of Spectral Decomposition Theorem (QSDT) which gives a direct connection with QEH and classical limit. In addition we consider the degree of generality of QSDT which make it useful as a framework for quantum chaos. All these aspects are in accordance with conceptual foundations in context of Belot-Earman program 8. Other relevant consequences of QSDT are: A characterization of first two levels of QEH (see theorem 10 of section 4.2) plus a simple connection between these levels and quantum spectrum in the classical limit (see section 5). In section 2 we begin introducing a brief review of minimal notions of density theory for the development of following sections.

\section{Theory of densities and Markov Operators}

Historically, the concept of density has only recently appeared in order to unify descriptions of phenomena of statistical nature. Clear examples of this are Maxwell velocity distribution and quantum mechanics, considered as attempts to unify theory of gases and as justification for the derivation of Planck distribution of black body radiation respectively.

Moreover, development of modern physics demonstrated the usefulness of densities to give a description of systems with a large number of freedom degree which have an uncertainty by ignorance. In this section we introduce a brief review of theory of densities and Markov operators based on dynamical systems formalism [1].

\subsection{Density functions and Dynamical Systems}

We begin recalling mathematical elements of dynamical systems theory. As we mentioned in introduction, these elements and definitions can be given within the framework of measurement theory [1, 11. Given a set $X, \Sigma$ is a $\sigma$-algebra of subsets of $X$ if it satisfies:

(I) $X \in \Sigma$

(II) $A, B \in \Sigma \Longrightarrow A \backslash B \in \Sigma$

$$
\left(B_{i}\right) \in \Sigma \Longrightarrow \cup_{i} B_{i} \in \Sigma \Theta
$$

A function $\mu$ on $\Sigma$ is a probability measure if it satisfies:

(I) $\mu: \Sigma \rightarrow[0,1]$ and $\mu(X)=1$

\footnotetext{
${ }^{3}$ In this paper this will be our mathematical framework.

${ }^{4}$ Index $i$ must run out a countable set.
} 
(II) For all countable family of pairwise disjoint subsets $\left(B_{i}\right) \in \Sigma \Longrightarrow \mu\left(\cup_{i} B_{i}\right)=\sum_{i} \mu\left(B_{i}\right)$

A measure space is any shortlist of the form $(X, \Sigma, \mu)$. Given a measure space $(X, \Sigma, \mu)$, a measure preserving transformation or automorphism $T$ is a function $T: X \rightarrow X$ which satisfies:

$$
\forall A \in \Sigma: \mu\left(T^{-1} A\right)=\mu(A)
$$

Then we say that family of transformations $\tau:=\left\{T_{t}: X \rightarrow X\right\}_{t \in \mathbb{R}}$ satisfying Eq. (1) and (see definition 7.2.1. of [1])

(a) $T_{0}(x)=x$ for all $x \in X$

(b) $T_{t_{1}}\left(T_{t_{2}}(x)\right)=T_{t_{1}+t_{2}}(x)$ for all $x \in X$ and $t_{1}, t_{2} \in \mathbb{R}$

(c) The mapping $(t, x) \rightarrow T_{t}(x)$ from $\mathbb{R} \times X$ into $X$ is continuous.

is a group of measure preserving automorphisms and we call it a dynamical law $\tau$. With these definitions, we say that quaternary $(X, \Sigma, \mu, \tau)$ is a dynamical system.

In dynamical system theory the central notion is the definition of density: Given a dynamical system $(X, \Sigma, \mu, \tau)$ and $D(X, \Sigma, \mu)=\left\{f \in L^{1}(X, \Sigma, \mu): f \geq 0 ;\|f\|=1\right\}$ any function $f \in D(X, \Sigma, \mu, \tau)$ is called a density.

In classical mechanics it is usual to take $X=\mathcal{M}$ as phase space, $\Sigma=\mathcal{P}(\mathcal{M})$ as power set of phase space, $\mu$ as Lebesgue measure and $T_{t}$ as time evolution transformation governed by Hamilton equations. This context will be clarified in the next sections. In addition to dynamical system definition other fundamental concept is the notion of Markov operator. This important class of operators is presented below.

\subsection{Markov Operators}

Given a classical system $S$ with an initial state given by a density $f_{0}$ we know that its temporal evolution will be determined by Liouville equation. Except in simple cases we know that this equation has no exact solution and thus we are forced to use another strategy to study the evolution of system. In this sense Markov operators are very useful because their properties allow us to know the asymptotic behavior of densities. General behavior of densities can be well developed in both dynamical and stochastic systems. Markov operators contain global information concerning densities in the asymptotic limit $t \rightarrow \infty$. Under certain hypotheses on Markov operators we have conditions for existence of an equilibrium density $f_{*}$ which physically corresponds to equilibrium approach. The approach to equilibrium in the limit $t \rightarrow \infty$ by means of Markov operators will be the link between classical limit and Quantum Spectral Decomposition Theorem. This will be considered in section 4 . We present a brief review of minimal concepts for the development of this paper beginning with the following definition (see [1], pag. 32).

Definition 2.1. (Markov Operator) Given a measure space $(X, \Sigma, \mu)$ a linear operator $P$ : $L^{1} \rightarrow L^{1}$ is called a Markov operator if it satisfies:

(a) Pf $\geq 0$

\footnotetext{
${ }^{5}$ In the general case $\tau$ is required to be a semigroup, i.e. in semidynamical systems (see definition 7.2.3 of 11). In dynamical systems $\tau$ is a group while in semidynamical systems $\tau$ is required to be a semigroup. Therefore, the dynamical systems are invertible while the semidynamical systems may not be invertible.
} 
(b) $\|P f\|=\|f\|$

for all $f \in L^{1}, f \geq 0$

From condition (b) it follows that $P$ is monotonic, that is if $f, g \in L^{1}$ with $f \geq g$ then $P f \geq P g$. Markov operators satisfy important properties that will be crucial in the derivation of a quantum version of Spectral Decomposition Theorem (see [1, pag. 33).

Theorem 1. Let $(X, \Sigma, \mu)$ be a $\sigma$-algebra and let $f \in L^{1}$. If $P$ is a Markov operator then:

(I) $\|P f\| \leq\|f\|$ (contractive property)

(II) $|P f(x)| \geq P|f(x)|$

The notion of fixed point of a Markov operator is fundamental to establishing the approach to equilibrium of a density (see [1], pag. 35).

Definition 2.2. (Fixed Point) Let $P$ be a Markov operator. If $f \in L^{1}$ with $P f=f$ then $f$ is called a fixed point of $P$. In a more general way, any $f \in D(X, \Sigma, \mu)$ that satisfies $P f=f$ is called a stationary density of $P$.

A family of automorphisms $\left\{T_{t}\right\}_{t \in \mathbb{R}}$ representing the evolution of any dynamical system is a special class of Markov operators called Frobenius-Perron operators. They are defined as follows (see [1], pag. 36).

Definition 2.3. (Frobenius-Perron Operator) Given a measure space $(X, \Sigma, \mu)$ and $T: X \rightarrow X$ a non singular automorphism (i.e. $\mu\left(T^{-1}(A)\right)=0$ for all $A \in \Sigma$ such that $\mu(A)=0$ ) the unique operator $P: L^{1} \rightarrow L^{1}$ defined for all $A \in \Sigma$ by the equation

$$
\int_{A} \operatorname{Pf}(x) \mu(d x)=\int_{T^{-1}(A)} f(x) \mu(d x)
$$

is called the Frobenius-Perron operator corresponding to T.

From Eq. (2) we have that Frobenius-Perron operator is linear and has the following properties (see [1], pag. 37).

Theorem 2. Let $T$ be an automorphism. If $P$ and $P_{n}$ are the Frobenius-Perron operators corresponding to $T$ and $T^{n}$ respectively. Then we have that

(I) $\int_{X} \operatorname{Pf}(x) \mu(d x)=\int_{X} f(x) \mu(d x)$

(II) $P_{n}=P^{n}$

The adjoint of a Frobenius-Perron operator is defined as follows (see [1], pag. 42).

Definition 2.4. (Koopman Operator) Given a measure space $(X, \Sigma, \mu)$ and $T: X \rightarrow X$ a non singular automorphism, the unique operator $U: L^{\infty} \rightarrow L^{\infty}$ defined for all $f \in L^{\infty}$ by

$$
U f(x)=f(T(x))
$$

is called the Koopman operator corresponding to $T$. 
From Eq. (3) we have that Koopman operator is linear and has the following properties (see [1], pag. 43):

Theorem 3. Let $T$ be an automorphism. If $U$ and $U_{n}$ are the Koopman operators corresponding to $T$ and $T^{n}$ respectively. Then

(I) $\|U f\|_{L^{\infty}} \leq\|f\|_{L^{\infty}}$

(II) $U_{n}=U^{n}$

$$
\left\langle P_{n} f, g\right\rangle=\left\langle f, U_{n} g\right\rangle \quad \text { for every } f \in L^{1}, g \in L^{\infty}, n \in \mathbb{N}_{0}
$$

where $\langle f, g\rangle=\int_{X} f(x) g(x) \mu(d x)$ for all $f \in L^{1}, g \in L^{\infty}$.

Now we recall the Ergodic Hierarchy of dynamical systems (see [1, pag. 66).

Theorem 4. (Ergodic, Mixing and Exact) Let $(X, \Sigma, \mu)$ be a normalized measure space, $T$ : $X \rightarrow X$ an automorphism and $P, U$ the Frobenius-Perron and Koopman operators corresponding to T. Then:

(a) $T$ is ergodic $\Leftrightarrow \lim _{n \rightarrow \infty} \frac{1}{n} \sum_{k=0}^{n}\left\langle P^{k} f, g\right\rangle=\lim _{n \rightarrow \infty} \frac{1}{n} \sum_{k=0}^{n}\left\langle f, U_{k} g\right\rangle=\langle f, 1\rangle\langle 1, g\rangle$ for all $f \in L^{1}, g \in L^{\infty}$

(b) $T$ is mixing $\Leftrightarrow \lim _{n \rightarrow \infty}\left\langle P^{n} f, g\right\rangle=\lim _{n \rightarrow \infty}\left\langle f, U_{n} g\right\rangle=\langle f, 1\rangle\langle 1, g\rangle$ for all $f \in L^{1}, g \in L^{\infty}$

(c) $T$ is exact $\Leftrightarrow \lim _{n \rightarrow \infty}\left\|P^{n} f-\langle f, 1\rangle\right\|=0 \quad$ for all $f \in L^{1}$

From these definitions (see [1] pag. 73) it follows that 0

$$
E X A C T \Longrightarrow M I X I N G \Longrightarrow E R G O D I C
$$

It should be noted that levels are substantially different from each other $\square$. To end this section we introduce the notion of constrictive operator which allow us to ensure the existence of an equilibrium density (see [1], pag. 87).

Definition 2.5. (constrictive operator) A Markov operator $P$ will be called constrictive if there exist a precompact set $\mathcal{F} \subseteq L^{1}$ such that for all $f \in D(X, \Sigma, \mu)$ :

$$
\lim _{n \rightarrow \infty} d\left(P^{n} f, \mathcal{F}\right)=\lim _{n \rightarrow \infty} i n f_{g \in \mathcal{F}}\left\|P^{n} f-g\right\|=0
$$

A relevant result is that every Markov constrictive operator has an equilibrium density (see [1], pag. 87).

\footnotetext{
${ }^{6}$ Ergodic, mixing and exact correspond to Cèsaro limit, weak limit and strong limit respectively. The terms "Cèsaro", "weak" and "strong" indicate the type of convergence of sequence $\left\{P^{n} f\right\}$ to 1 (see theorem 4.4.1 of [1]).

${ }^{7}$ A full distinction between ergodic, mixing and exact is illustrated by first six successive iterates of a random distribution of 1000 points in $X=[0,1] \times[0,1]$ with the corresponding transformations $S_{\text {ergodic }}(x, y)=(\sqrt{2}+$ $x, \sqrt{3}+y)(\bmod 1), S_{\text {mixing }}(x, y)=(x+y, x+2 y)(\bmod 1)$ and $S_{\text {exact }}(x, y)=(3 x+y, x+3 y)(\bmod 1) . \operatorname{In}$ each case the effect of transformation is to move around the space, spread throughout space and quickly spread throughout the space corresponding to the ergodic, mixing and exact levels respectively (see fig. 4.3.3, fig. 4.3.4 and fig. 4.3 .5 of [1]).
} 
Theorem 5. Let $(X, \Sigma, \mu)$ be a normalized measure space, and $P: L^{1} \rightarrow L^{1}$ a constrictive Markov operator. Then $P$ has a stationary density, i.e there is a $f_{*} \in L^{1}$ such that $P f_{*}=f_{*}$.

The existence of equilibrium densities can be considered relevant to obtain a theoretical framework for quantum chaos. For instance, in quantum billiards the statement of quantum ergodicity is equivalent to the statement of uniform equidistribution of probability density for eigenstates. Moreover, this fact can be translated to quantum language through Wigner transform such that equilibrium state of any closed quantum system with continuous spectrum is represented by the weak limit $\hat{\rho}_{*}$ (see [26], pag. 889 eq. (3.28)).

\section{The Spectral Decomposition Theorem of Dynamical Systems}

With all the mathematical background of previous section we are able to present one of the main results of dynamical systems theory called the Spectral Decomposition Theorem (SDT) (see 11, pag. 88).

Theorem 6. (The Spectral Decomposition Theorem(version I)) Let P be a Markov constrictive operator. Then there is an integer $r$, two sequences of nonnegative functions $g_{i} \in D(X, \Sigma, \mu)$, $k_{i} \in L^{\infty}, i=1, \ldots, r$, and an operator $Q: L^{1} \rightarrow L^{1}$ such that for all $f \in L^{1}$, Pf may be written as

$$
P f(x)=\sum_{i=1}^{r} \lambda_{i}(f) g_{i}(x)+Q f(x)
$$

where

$$
\lambda_{i}(f)=\int_{X} f(x) k_{i}(x) \mu(d x)=\left\langle f(x), k_{i}(x)\right\rangle
$$

The functions $g_{i}$ and the operator $Q$ have the following properties:

(I) $g_{i}(x) g_{j}(x)=0$ for all $i \neq j$, so that functions $g_{i}$ have disjoint supports.

(II) For each integer $i$ there exists a unique integer $\alpha(i)$ such that $P g_{i}=g_{\alpha(i)}$. Further $\alpha(i) \neq$ $\alpha(j)$ for $i \neq j$ thus the operator $P$ just permutes the functions $g_{i}$.

$$
\left\|P^{n} Q f\right\| \rightarrow 0 \quad \text { as } \quad n \rightarrow \infty \text { for every } f \in L^{1} \text {. }
$$

Assuming that time evolution has a constrictive Perron-Frobenius operator then Eq. (6) describes the evolution of any density having an initial term which oscillates between densities $g_{i}$. We note that $Q f$ is associated with a relaxation process which we will analyze in section 5 . By property (II) of Theorem (마) we have

$$
P^{n} f(x)=\sum_{i=1}^{r} \lambda_{i}(f) g_{\alpha^{n}(i)}(x)+P^{n-1} Q f(x)=\sum_{i=1}^{r} \lambda_{\alpha^{-n}(i)}(f) g_{i}(x)+Q_{n} f(x)
$$

where $Q_{n} f(x)=P^{n-1} Q f(x)$ and $\left\{\alpha^{-n}(i)\right\}$ is the inverse permutation of $\left\{\alpha^{n}(i)\right\}$. When the measure space is normalized and Markov operator $P$ has a constant stationary density $f_{*}$ (e.g., if $\mathrm{P}$ is a Frobenius-Perron operator this is equivalent to $\mu_{f}$ invariant, see [1], pag. 46) Spectral Decomposition Theorem takes the following compact form (see [1, pag. 90). 
Theorem 7. (Version (II) of The Spectral Decomposition Theorem) Let $(X, \Sigma, \mu)$ be a normalized measure space and $P: L^{1} \rightarrow L^{1}$ a constrictive Markov operator. If $P$ has a stationary density then the representation of $P^{n} f$ takes the simple form for all $f \in L^{1}$

$$
P^{n} f(x)=\sum_{i=1}^{r} \lambda_{\alpha^{-n}(i)}(f) \overline{1}_{A_{i}}(x)+Q_{n} f(x)
$$

where

$$
\begin{gathered}
\overline{1}_{A_{i}}(x)=\left[1 / \mu\left(A_{i}\right)\right] 1_{A_{i}} \\
\bigcup_{i} A_{i}=X \quad \text { with } \quad A_{i} \cap A_{j}=\emptyset \quad \text { for } \quad i \neq j
\end{gathered}
$$

and 8

$$
\mu\left(A_{i}\right)=\mu\left(A_{j}\right) \text { if } j=\alpha^{n}(i) \text { for some } n .
$$

Theorem (77) characterizes the Ergodic Hierarchy levels by means of permutation $\left\{\alpha^{n}(i)\right\}$ (see [1], pag. $92-94)$.

Theorem 8. (The Spectral Decomposition Theorem and Ergodic Hierarchy) Let $(X, \Sigma, \mu)$ be a normalized measure space and $P: L^{1} \rightarrow L^{1}$ a constrictive Markov operator. Then

(I) $P$ is ergodic $\Longleftrightarrow$ permutation $\{\alpha(1), \ldots, \alpha(r)\}$ of sequence $\{1, \ldots, r\}$ is cyclical (that is, for which there no is invariant subset).

(II) If $r=1$ in representation of $E q .(6) \Longrightarrow P$ is exact.

(III) If $P$ is mixing $\Longrightarrow r=1$ in representation of Eq. (6).

It should be noted that, under hypothesis of Theorem 8, $P$ mixing implies $P$ exact. Then since $P$ exact implies $P$ mixing (see eq. (4)) it follows that $P$ exact $\Longleftrightarrow P$ mixing $\Longleftrightarrow r=1$ (see remark 5.5.1 of [1]).

Theorem (7) says that if classical Hamiltonian is such that Frobenius-Perron operator $P$ associated to time evolution $T$ admits an equilibrium density $f_{*}$ then in the asymptotic limit $(n \rightarrow \infty)$ the state of the system $U_{n} f(x)=f\left(T_{n}(x)\right)$ will oscillate between characteristic functions $\overline{1}_{A_{i}}(x)$ with a remainder term $Q_{n} f(x)$ going to zero. In next section we will see that a quantum version of SDT (called QSDT) contains relevant information about quantum spectrum. We have seen that constrictiveness of a Markov operator $P$ and normalization of measure space are sufficient to ensure the existence of stationary densities and to obtain a representation of their time evolution by means of SDT.

\footnotetext{
${ }^{8}$ In equations (9)-(12) the index $i$ runs over a countable set.
} 


\section{The Quantum Version of Spectral Decomposition Theorem (QSDT)}

The aim of this paper is to obtain a quantum version of SDT, called the Quantum Version of Spectral Decomposition Theorem (QSDT), that can be useful to study quantum systems in the classical limit and to give an alternative framework for quantum chaos according to Berry's definition. In this section we begin by defining the mathematical concepts of this approach considering the observables as central objects and the states as functionals of those. In order to apply QSDT to open quantum systems (see section 6) we consider the quantum characteristic algebra $\mathcal{A}$ whose elements $\hat{O} \in \mathcal{A}$ are observables not necessarily self-adjoint. In other words, Hermiticity condition $\hat{O}=\hat{O}^{\dagger}$ can be relaxed and this is motivated for several reasons some of them we can list below:

(a) The interest in the study of non-Hermitian Hamiltonians related with the interpretation of some properties such as transfer phenomena, nuclear resonances, typical of open systems.

(b) In scattering systems one can consider quantum resonances, i.e. "quasi-stationary states" instead of scattering solutions [27]. These resonances can play a similar role in open systems as eigenstates in closed systems and their eigenvalues are complex numbers with non zero imaginary part, see sections 5.1.1. and 6 .

(c) Any measurement on a wave system drastically changes its properties by converting discrete energy levels into decaying resonances called "quasi-stationary states" which can be described by a non-Hermitian Hamiltonian [28, 29, 30]. This situation will be illustrated in section 6.

(d) With the purpose to take into account non-unitary time evolutions that appear in descriptions of open quantum systems the introduction of non-Hermitian observables becomes "natural" and fundamental from a theoretical viewpoint.

(e) Non-Hermitian operators are frequently used to mathematically represent potentials of open quantum systems describing ionization or dissociation where the system breaks up into freely moving non-interacting subsystems, see pag. 4 of [30].

(f) The complex expectation value $A_{\hat{O}}=\langle\hat{O}\rangle=\left|A_{\hat{O}}\right| e^{i \alpha}$ of a non-Hermitian observable $\hat{O}$ can be physically interpreted postulating that the absolute value $\left|A_{\hat{O}}\right|$ and the phase $\alpha$ are measurable quantities, see pag. 11 of [30].

We point out that conclusions obtained from QSDT will be valid for both closed and open quantum systems. However, in section 6 we will only illustrate QSDT with examples of open quantum systems in the research ling of [31] which states: "The approach of linking chaos with the unpredictability of the measurement outcomes is the right one in the quantum case". In this paper we will not discuss this entropic approach of quantum chaos but it should be noted that

\footnotetext{
${ }^{9}$ We mean that examples presented in this paper belong theoretically to the category of quantum systems within the approach of [31, i.e. open quantum systems which are disturbed by the measuring process like microwave billiards, etc.

${ }^{10}$ The term "intrinsic" refers to quantum dynamical of closed quantum systems described by wave functions of integrable square and thus quantum evolution becomes almost periodic at least for the finite dimensional case. In contrast, the term "genuine" is associated with the approach based on that successive outcomes of a measurement can form chaotic and unpredictable sequences 31 .
} 
closed quantum systems $\Longrightarrow$ hermitian observables $\Longrightarrow$ "intrinsic quantum chaos"

open quantum systems $\Longrightarrow$ observables (herm. and non-herm.) $\Longrightarrow$ "genuine quantum chaos"

This distinction can be seem trivial and meaningless but it allows to place algebra of hermitian operators and unitary evolutions in the context of closed quantum systems. On the other hand, second line of Eq. (13) places algebra of operators (hermitian and non-hermitian) and evolutions (unitary and non-unitary) in the context of open quantum systems. Returning to the mathematical background, the space of states is the positive cone

$$
\mathcal{N}=\left\{\hat{\rho} \in \mathcal{A}^{\prime}: \hat{\rho}(\mathbb{I})=1, \quad \hat{\rho}^{\dagger}=\hat{\rho}, \quad \hat{\rho}\left(\hat{a} . \hat{a}^{\dagger}\right) \geq 0 \text { for all } \hat{a} \in \mathcal{A}\right\}
$$

where the action $\hat{\rho}(\hat{O})$ of functional $\hat{\rho} \in \mathcal{A}^{\prime}$ on the observable $\hat{O} \in \mathcal{A}$ is denoted by $(\hat{\rho} \mid \hat{O})$. In other words, $(\hat{\rho} \mid \hat{O})$ is the mean value of $\hat{O}$ in $\hat{\rho}$, i.e. $(\hat{\rho} \mid \hat{O})=\langle\hat{O}\rangle_{\hat{\rho}}=\operatorname{tr}(\hat{\rho} \hat{O})$. When $\hat{O}=\mathbb{I}$ this action is the trace of $\hat{\rho}$ which is equal to $\operatorname{tr}(\hat{\rho})=\hat{\rho}(\mathbb{I})=(\hat{\rho} \mid \mathbb{I})=1$. We note the dual of $\mathcal{A}$ as $\mathcal{A}^{\prime}$.

In this approach the state is unknown and we only focus in the study of expectation values $(\hat{\rho}(t) \mid \hat{O})$ for large times $t \rightarrow \infty$. If for all $\hat{\rho} \in \mathcal{A}^{\prime}$ there exists a unique $\hat{\rho}_{*} \in \mathcal{A}^{\prime}$ such that

$$
\lim _{t \rightarrow \infty}(\hat{\rho}(t) \mid \hat{O})=\lim _{t \rightarrow \infty}\left(\hat{U}_{t} \hat{\rho} \hat{U}_{t}^{\dagger} \mid \hat{O}\right)=\left(\hat{\rho}_{*} \mid \hat{O}\right)
$$

we say that $\hat{\rho}$ has weak-limit $\hat{\rho}_{*}$ (see [2] pag. 248). Functional $\hat{\rho}_{*}$ is interpreted as the average value that would result if state $\hat{\rho}(t)$ had a limit $\hat{\rho}_{*}$ for $t \rightarrow \infty$. That is, $\hat{\rho}_{*}$ is a weak limit and not a limit in the (strong) usual sense. In other words $\hat{\rho}_{*}$ is an equilibrium state, in the weak sense, that system reaches in its process of relaxation.

Now it is suitable to make the following remark. In general, if evolution operator $\hat{U}_{t}$ is not unitary (for example when Hamiltonian is non-Hermitian, see section 6) then we will have that $0<\operatorname{tr}\left(\hat{\rho}_{*}\right)<1$, i.e. weak limit $\hat{\rho}_{*} \in \mathcal{A}^{\prime} \backslash \mathcal{N}$ is considered an state not normalized. This means that trace of any initial state $\hat{\rho}$ is not preserved for non-unitary evolutions in the limit $t \rightarrow \infty$. This is a typical feature of decoherence in open quantum systems where interaction between a quantum system and its environment produces a non-unitary evolution of reduced state described by a master equation [32].

A fundamental mathematical element to study the classical limit of a quantum system is the Wigner transform. From a quantum state $\hat{\rho}$ Wigner transform allows us to obtain a function $f(\phi)$ defined over phase space $\Gamma$ that can be interpreted, in the classical limit $\hbar \rightarrow 0$, as a distribution probability governed by Liouville equation of classical statistical mechanics. We present a brief review of some relevant properties of Wigner transform.

Let $\Gamma=\mathcal{M}_{2(N+1)} \equiv \mathbb{R}^{2(N+1)}$ be the phase space. Wigner transformation symb $: \mathcal{A} \rightarrow \mathcal{A}_{q}$ sends quantum algebra $\mathcal{A}$ to a "classical-like" $\mathcal{A}_{q}$ algebra by (see [33, 34, 35]).

$$
\operatorname{symb}(\hat{f})=f(\phi)=\int_{\Gamma}\langle q+\Delta|\hat{f}| q-\Delta\rangle e^{i \frac{p \Delta}{\hbar}} d^{N+1} \Delta
$$

where $f(\phi) \in \mathcal{A}_{q}$ are functions defined over space phase $\Gamma$ where $\phi=\left(q^{1}, \ldots, q^{N+1}, p_{q}^{1}, \ldots, p_{q}^{N+1}\right)$. The star product between two operators $\hat{f}, \hat{g} \in \mathcal{A}$ is given by (see [33])

$$
\operatorname{symb}(\hat{f} . \hat{g})=\operatorname{symb}(\hat{f}) * \operatorname{symb}(\hat{g})=(f * g)(\phi)
$$


and the Moyal bracket (see [35]) is

$$
\{f, g\}_{m b}=\frac{1}{i \hbar}(\operatorname{symb}(\hat{f}) * \operatorname{symb}(\hat{g})-\operatorname{symb}(\hat{g}) * \operatorname{symb}(\hat{f}))=\operatorname{symb}\left(\frac{1}{i \hbar}[\hat{f}, \hat{g}]\right)
$$

Two important properties are, see 33

$$
(f * g)(\phi)=f(\phi) g(\phi)+0(\hbar) \quad, \quad\{f, g\}_{m b}=\{f, g\}_{p b}+0\left(\hbar^{2}\right)
$$

The symmetrical or Weyl ordering prescription is used to define inverse map symb $b^{-1}$, that is

$$
\operatorname{symb}^{-1}\left[q^{i}(\phi), p^{j}(\phi)\right]=\frac{1}{2}\left(\hat{q}^{i} \hat{p}^{j}+\hat{p}^{j} \hat{q}^{i}\right)
$$

Therefore, symb and symb ${ }^{-1}$ define isomorphisms between the algebras $\mathcal{A}$ and $\mathcal{A}_{q}$,

$$
\text { symb }: \mathcal{A} \rightarrow \mathcal{A}_{q} \quad, \quad \text { symb }^{-1}: \mathcal{A}_{q} \rightarrow \mathcal{A}
$$

On the other hand, Wigner transformation for states is

$$
\rho(\phi)=(2 \pi \hbar)^{-(N+1)} \operatorname{symb}(\hat{\rho})
$$

The fundamental property of Wigner transform used throughout this paper is the preservation of inner product between states $\hat{\rho} \in \mathcal{N}$ and observables $\hat{O} \in \mathcal{A}$. From a physical viewpoint this property correspond to the invariance of mean values calculated in $\mathcal{A}$ and $\mathcal{A}_{q}$ respectively. More precisely,

$$
\hat{\rho}(\hat{O})=\langle\hat{O}\rangle_{\hat{\rho}}=(\hat{\rho} \mid \hat{O})=(\operatorname{symb}(\hat{\rho}) \mid \operatorname{symb}(\hat{O}))=\langle\rho(\phi), O(\phi)\rangle=\int_{\Gamma} d \phi^{2(N+1)} \rho(\phi) O(\phi)
$$

More generally, if $\hat{A}, \hat{B} \in \mathcal{A}$ then we have (see [33, 36])

$$
\operatorname{tr}(\hat{A} \hat{B})=(\hat{A} \mid \hat{B})=(\operatorname{symb}(\hat{A}) \mid \operatorname{symb}(\hat{B}))=\langle A(\phi), B(\phi)\rangle=\int_{\Gamma} d \phi^{2(N+1)} A(\phi) B(\phi)
$$

where $A(\phi)=(2 \pi \hbar)^{-(N+1)} \operatorname{symb}(\hat{A})$ and $B(\phi)=\operatorname{symb}(\hat{B})$.

At this point it is suitable to make the following remarks and clarifications.

(I) In this paper we use $\hat{\rho}(n)$ as a short notation of $\hat{\rho}$ after $n$ successive applications of $\hat{U}$, i.e. $\hat{\rho}(n)=\hat{U}(n) \hat{\rho} \hat{U}(n)^{\dagger}$ represents $\hat{\rho}$ after $n$ arbitrary time steps at constant intervals in a discretized time evolution. More precisely, we consider that $\hat{U}(n)$ is the one given by Hamiltonian or Floquet operator [4]. Both cases are of great interest theoretically and experimentally $\square$. That is, we can choose $\hat{U}$ as evolution operator and make the time step to be equal to $\alpha \in \mathbb{R}$ then we have $\hat{\rho}(n)=\left(e^{-i \frac{\hat{H}}{\hbar} \alpha n}\right) \hat{\rho}\left(e^{-i \frac{\hat{H}^{\dagger}}{\hbar} \alpha n}\right)$ where only in the unitary case we will have $\hat{H}^{\dagger}=\hat{H}$. Moreover, in applications of oscillating electric (or magnetic) fields we have Hamiltonians of the type $\hat{H}=\hat{H}_{0}+\hat{V}(t)$ where $\hat{H}_{0}$ is typically

\footnotetext{
${ }^{11}$ For instance, when we have Hamiltonians with periodic time dependences the theoreticians prefer periodically kicked systems while the experimentalists prefer driven systems (see pag. 138 and 139 of [4]).
} 
the Hamiltonian of an atom or nucleus with a time-periodic potential $\hat{V}(t)$. In this case the "natural" choice for $\hat{U}$ is the Floquet operator so that $\hat{\rho}(n)$ represents an stroboscopic observation of the system in $\hat{\rho}$ at time $t=n \tau$ where $\tau$ is the periodicity of $\hat{V}(t)$ (see section 4.1 of [4]). In section 5 we will take into account that the choice of time steps is arbitrary.

$(I I)$ By "a time evolution operator $\hat{U}$ having a classical evolution automorphism $T$ " we will mean that if $\hat{\rho}$ is a quantum state and $\rho=\operatorname{sym} b(\hat{\rho})$ is its corresponding density then Wigner transform of $\hat{\rho}(1)$ (i.e. $\hat{\rho}(1)$ is $\hat{\rho}$ after an application of $\hat{U})$ is $\operatorname{sym} b(\hat{\rho}(1))=\operatorname{symb}\left(\hat{U} \hat{\rho} \hat{U}^{\dagger}\right)=$ $\rho \circ T$. In other words, Wigner transform connects evolution operator $\hat{U}$ with automorphism $T$. Moreover, it follows that $\operatorname{symb}(\hat{\rho}(n))=\operatorname{symb}\left(\hat{U}(n) \hat{\rho} \hat{U}(n)^{\dagger}\right)=\rho \circ T^{n}$ for all $n \in \mathbb{N} \cup\{0\}$.

These remarks will be fundamental for the development of next sections.

\subsection{The Quantum Spectral Decomposition Theorem (QSDT)}

In previous section we presented a framework based on the classical limit by means of Wigner transform. Now we can write Spectral Decomposition Theorem (Theorem 7 of section 3) in quantum language. First, we assume that

- If $\hat{U}$ represents the evolution[ᄑ] of a quantum system then $\hat{U}$ has a corresponding classical evolution automorphism $13 T$ defined over $\Gamma$ and $T$ has an associated constrictive Frobenius-Perron operator $P$.

- There exists a stationary density $f_{*}$, that is, $P f_{*}=f_{*}$.

Under these hypothesis we have the following quantum version of Spectral Decomposition Theorem.

Theorem 9. (The Quantum Spectral Decomposition Theorem(QSDT)) Let $\hat{\rho} \in \mathcal{N}$ and let $\hat{O}$ be an observable. Then there exists pure states $\hat{\rho}_{1}, \hat{\rho}_{2}, \ldots, \hat{\rho}_{r} ;$ observables $\hat{O}_{1}, \hat{O}_{2}, \ldots, \hat{O}_{r} ; a$ permutation $\alpha:\{1, \ldots, r\} \longrightarrow\{1, \ldots, r\}$ and $\widetilde{\rho}_{0} \in \mathcal{A}^{\prime}$ such that

$$
(\hat{\rho}(n) \mid \hat{O})=\sum_{i=1}^{r} \lambda_{\alpha^{-n}(i)}\left(\hat{\rho}_{i} \mid \hat{O}\right)+\left(\widetilde{\rho}_{0}(n-1) \mid \hat{O}\right)
$$

where ${ }^{14}$

$$
\begin{aligned}
& \hat{\rho}(n)=U(n) \hat{\rho} U(n)^{\dagger} \text { and } \\
& \lambda_{i}(\hat{\rho})=\left(\hat{\rho} \mid \hat{O}_{i}\right)
\end{aligned}
$$

The states $\hat{\rho}_{i}$ and $\widetilde{\rho}_{0}$ have the following properties:

\footnotetext{
${ }^{12}$ By remark $(I)$ of previous section we know that $\hat{U}$ is not necessarily $\hat{U}=e^{-i \frac{\hat{H}}{\hbar} t}$ given by Hamiltonian, i.e. $\hat{U}$ may also be Floquet operator.

${ }^{13}$ Of course, we can make the natural choice of $T$ as $T: \Gamma \rightarrow \Gamma$ with $T(\phi=(q, p))=\phi(1)=(q(1), p(1))$, i.e. time step is equal to one and $T$ is the classical evolution operator determined by Hamilton equations. However, the choice of time steps is arbitrary.

${ }^{14} \hat{\rho}(n)$ in the sense of the remark $(I)$ of pag. 11.
} 
(I) $\hat{\rho}_{i} \hat{\rho}_{j}=0(\hbar)$ for all $i \neq j$ and $\hat{\rho}_{i}^{2}=\hat{\rho}_{i}+0(\hbar)$. So that states $\hat{\rho}_{i}$ are projectors in the classical limit $(\hbar \rightarrow 0)$. Moreover, we have a decomposition of the identity:

$$
\hat{1}=\sum_{i} \alpha_{i} \hat{\rho}_{i} \quad \text { with } \quad \alpha_{i} \geq 0, \sum_{i} \alpha_{i}=1
$$

(II) For each integer $i$ there exists a unique integer $\alpha(i)$ such that $\left(\hat{U} \hat{\rho}_{i} \hat{U}^{\dagger} \mid \hat{O}\right)=\left(\hat{\rho}_{\alpha(i)} \mid \hat{O}\right)$. Further $\alpha(i) \neq \alpha(j)$ for $i \neq j$ so operator $\hat{U}$ permutes the states $\hat{\rho}_{i}$.

$(I I I)\left(\widetilde{\rho}_{0}(n-1) \mid \hat{O}\right) \longrightarrow 0 \quad$ as $\quad n \longrightarrow \infty$.

Proof. We consider that triplet $(X, \Sigma, \mu)$ is the one given by classical mechanics, i.e $X=\Gamma$ is the $2(N+1)$-dimensional phase space, $\Sigma=\mathcal{P}(\Gamma)$ is the power set of $\Gamma$ and $\mu$ is the Lebesgue measure. As is usual, we denote $x \in X$ by $\phi=\left(q^{1}, \ldots, q^{N+1}, p_{q}^{1}, \ldots, p_{q}^{N+1}\right) \in \Gamma$ and $\mu(d x)$ by $d^{2(N+1)} \phi$. Let $\hat{\rho} \in \mathcal{N}$ and let $\hat{O}$ be an observable. If we define $f=\operatorname{symb}(\hat{\rho})$ and $g=\operatorname{symb}(\hat{O})$ then, multiplying Eq. (9) by $g$ and integrating over all phase space $\Gamma$ we have

$$
\int_{\Gamma} d^{2(N+1)} \phi P^{n} f(\phi) g(\phi)=\sum_{i=1}^{r} \lambda_{\alpha^{-n}(i)}(f) \int_{\Gamma} d^{2(N+1)} \phi \overline{1}_{A_{i}}(\phi) g(\phi)+\int_{\Gamma} d^{2(N+1)} \phi Q_{n} f(\phi) g(\phi)
$$

Equivalently,

$$
\left\langle P^{n} f, g\right\rangle=\sum_{i=1}^{r} \lambda_{\alpha^{-n}(i)}(f)\left\langle\overline{1}_{A_{i}}, g\right\rangle+\left\langle P^{n-1} Q f, g\right\rangle
$$

Since $\left\langle P^{n} f, g\right\rangle=\left\langle f, U^{n} g\right\rangle$ and $\left\langle P^{n-1} Q f, g\right\rangle=\left\langle Q f, U^{n-1} g\right\rangle$ (i.e. Koopman operator $U$ is the dual of Frobenius-Perron operator $P$ both corresponding to automorphism $T$ ) we obtain

$$
\left\langle f, U^{n} g\right\rangle=\sum_{i=1}^{r} \lambda_{\alpha^{-n}(i)}(f)\left\langle\overline{1}_{A_{i}}, g\right\rangle+\left\langle Q f, U^{n-1} g\right\rangle
$$

Now if we call $\hat{\rho}_{i}=s y m b^{-1}\left(\overline{1}_{A_{i}}\right), \widetilde{\rho}_{0}=\operatorname{symb}^{-1}(Q f)$ and use that $U^{n} g=g \circ T^{n}=g(n)=$ $\operatorname{symb}(\hat{O}(n)), U^{n-1} g=g \circ T^{n-1}=g(n-1)=\operatorname{symb}(\hat{O}(n-1))$ (see Eq. (3) $)$ then

$$
\langle\operatorname{symb}(\hat{\rho}), \operatorname{symb}(\hat{O}(n))\rangle=\sum_{i=1}^{r} \lambda_{\alpha^{-n}(i)}(f)\left\langle\operatorname{symb}\left(\hat{\rho}_{i}\right), \operatorname{symb}(\hat{O})\right\rangle+\left\langle\operatorname{symb}\left(\widetilde{\rho}_{0}\right), \operatorname{symb}(\hat{O}(n-1))\right\rangle
$$

If we call $k_{i}=\operatorname{symb}\left(\hat{O}_{i}\right)$ and use Eq. (7) then coefficient $\lambda_{\alpha^{-n}(i)}(f)$ can be written as

\footnotetext{
${ }^{15}$ Throughout the demonstration we consider that $T$ is an arbitrary automorphism and not necessarily the classical evolution $T_{t=1}(\phi=(q, p))=\phi(1)=(q(1), p(1))$ given by the Hamiltonian equations.
} 


$$
\lambda_{\alpha^{-n}(i)}(f)=\int_{\Gamma} d^{2(N+1)} \phi f(\phi) k_{\alpha^{-n}(i)}(\phi)=\left\langle f, k_{\alpha^{-n}(i)}\right\rangle=\left\langle\operatorname{symb}(\hat{\rho}), \operatorname{symb}\left(\hat{O}_{\alpha^{-n}(i)}\right)\right\rangle=\lambda_{\alpha^{-n}(i)}(\hat{\rho})
$$

Therefore, Eq. (31) reads

$$
\langle\operatorname{symb}(\hat{\rho}), \operatorname{symb}(\hat{O}(n))\rangle=\sum_{i=1}^{r} \lambda_{\alpha^{-n}(i)}(\hat{\rho})\left\langle\operatorname{symb}\left(\hat{\rho}_{i}\right), \operatorname{symb}(\hat{O})\right\rangle+\left\langle\operatorname{symb}\left(\widetilde{\rho}_{0}\right), \operatorname{symb}(\hat{O}(n-1))\right\rangle
$$

Finally, we can use the property of Wigner transform given by Eq. (23)

$$
\forall \hat{O} \in \mathcal{A}, \forall \hat{\rho} \in \mathcal{A}^{\prime}:(\hat{\rho} \mid \hat{O})=\langle\operatorname{symb}(\hat{\rho}), \operatorname{symb}(\hat{O})\rangle=\int_{\Gamma} d^{2(N+1)} \phi \rho(\phi) O(\phi)
$$

Using this property Eq. (33) can be expressed in quantum language as

$$
(\hat{\rho} \mid \hat{O}(n))=\sum_{i=1}^{r} \lambda_{\alpha^{-n}(i)}(\hat{\rho})\left(\hat{\rho}_{i} \mid \hat{O}\right)+\left(\widetilde{\rho}_{0} \mid \hat{O}(n-1)\right)
$$

We know that $(\hat{\rho} \mid \hat{O}(n))$ and $\left(\widetilde{\rho}_{0} \mid \hat{O}(n-1)\right)$ are equal to $(\hat{\rho}(n) \mid \hat{O})$ and $\left(\widetilde{\rho}_{0}(n-1) \mid \hat{O}\right)$ respectively, and then Eq. (35) reads as

$$
(\hat{\rho}(n) \mid \hat{O})=\sum_{i=1}^{r} \lambda_{\alpha^{-n}(i)}(\hat{\rho})\left(\hat{\rho}_{i} \mid \hat{O}\right)+\left(\widetilde{\rho}_{0}(n-1) \mid \hat{O}\right)
$$

Hence we have proved Eq. (25).

(I): We have

$$
\begin{aligned}
& \operatorname{tr}\left(\hat{\rho}_{i}\right)=\left(\hat{\rho}_{i} \mid \hat{1}\right)=\left\langle\operatorname{symb}\left(\hat{\rho}_{i}\right), \operatorname{symb}(\hat{1})\right\rangle=\left\langle\overline{1}_{A_{i}}, 1_{X}\right\rangle=\int_{\Gamma} d^{2(N+1)} \phi \overline{1}_{A_{i}}(\phi)=\int_{\Gamma} d^{2(N+1)} \phi\left[1 / \mu\left(A_{i}\right)\right] 1_{A_{i}}(\phi) \\
& =\left[1 / \mu\left(A_{i}\right)\right] \int_{\Gamma} d^{2(N+1)} \phi 1_{A_{i}}(\phi)=\left[1 / \mu\left(A_{i}\right)\right] \int_{A_{i}} d^{2(N+1)} \phi=\left[1 / \mu\left(A_{i}\right)\right] \mu\left(A_{i}\right)=1
\end{aligned}
$$

where we have used definition of $\overline{1}_{A_{i}}$ given by Eq. (10), the Weyl symbol property (see Eq. (34)) and that $\operatorname{symb}(\hat{1})=1_{X}$. Thus $\operatorname{tr}\left(\hat{\rho}_{i}\right)=1$, i.e. $\hat{\rho}_{i} \in \mathcal{N}$ for all $i$. On the other hand if we apply Eq. (19) to $f=\overline{1}_{A_{i}}$ and $g=\overline{1}_{A_{j}}$ we have

$$
\begin{aligned}
& \operatorname{symb}\left(\hat{\rho}_{i} \hat{\rho}_{j}\right)=\overline{1}_{A_{i}}(\phi) \overline{1}_{A_{j}}(\phi)+0(\hbar)= \\
& =0(\hbar) \text { if } i \neq j \\
& =\overline{1}_{A_{i}}(\phi)+0(\hbar) \quad \text { if } i=j
\end{aligned}
$$

Now applying $s y m b^{-1}$ to both sides of Eq. (38) we obtain 


$$
\begin{aligned}
& \hat{\rho}_{i} \hat{\rho}_{j}=0(\hbar) \quad \text { if } i \neq j \\
& =\hat{\rho}_{i}+0(\hbar) \quad \text { if } \quad i=j
\end{aligned}
$$

On the other hand from Eqns. (10) and (11) we have $1_{X}=\sum_{i} \mu\left(A_{i}\right) \overline{1}_{A_{i}}$ and therefore symb $^{-1}\left(1_{X}\right)=\operatorname{symb}^{-1}\left(\sum_{i} \mu\left(A_{i}\right) \overline{1}_{A_{i}}\right)=\sum_{i} \mu\left(A_{i}\right)$ symb $^{-1}\left(\overline{1}_{A_{i}}\right)$ where we have used that symb $b^{-1}$ is a linear map. Now if we call $\alpha_{i}=\mu\left(A_{i}\right)$ since $s y m b^{-1}\left(1_{X}\right)=\hat{1}$ and $\hat{\rho}_{i}=\operatorname{symb}^{-1}\left(\overline{1}_{A_{i}}\right)$ then we obtain Eq. (27).

(II): Due to part (II) of Theorem 6 and taking into account that we are working under hypothesis of Theorem 7 we have

$$
P \overline{1}_{A_{i}}=\overline{1}_{A_{\alpha}(i)}
$$

where $\alpha:\{1, \ldots, r\} \longrightarrow\{1, \ldots, r\}$ is a permutation which satisfies $\alpha(i) \neq \alpha(j)$ for $i \neq j$ and thus operator $P$ permutes the functions $\overline{1}_{A_{i}}$. Let $\hat{O}$ be an observable and $g=\operatorname{symb}(\hat{O})$. Then from Eq. (40) we have

$$
\left\langle P \overline{1}_{A_{i}}, g\right\rangle=\left\langle\overline{1}_{A_{\alpha}(i)}, g\right\rangle
$$

and noting that

$$
\begin{aligned}
& \left\langle P \overline{1}_{A_{i}}, g\right\rangle=\left\langle\overline{1}_{A_{i}}, U g\right\rangle=\left\langle\operatorname{symb}\left(\hat{\rho}_{i}\right), \operatorname{symb}(\hat{O}(1))\right\rangle=\left(\hat{\rho}_{i} \mid \hat{O}(1)\right)=\left(\hat{U} \hat{\rho}_{i} \hat{U}^{\dagger} \mid \hat{O}\right) \\
& \left\langle\overline{1}_{A_{\alpha}(i)}, g\right\rangle=\left\langle\operatorname{symb}\left(\hat{\rho}_{\alpha(i)}\right), \operatorname{symb}(\hat{O})\right\rangle=\left(\hat{\rho}_{\alpha(i)} \mid \hat{O}\right)
\end{aligned}
$$

then from Eq. (40) we have that $\left(\hat{U} \rho_{i} \hat{U}^{\dagger} \mid \hat{O}\right)=\left(\hat{\rho}_{\alpha(i)} \mid \hat{O}\right)$.

(III): Let $\hat{O}$ be an observable and $\varepsilon>0$. Then by condition (III) of Theorem 6 we have

$$
\left\|P^{n-1} Q f\right\|=\left\|Q_{n} f\right\|<\frac{\varepsilon}{\max \{|O(\phi)|: \phi \in \Gamma\}}=\frac{\varepsilon}{\|O\|_{\infty}} \quad \text { with } O=\operatorname{symb}(\hat{O})
$$

Then

$$
\left(\widetilde{\rho}_{0}(n-1) \mid \hat{O}\right)=\left\langle\operatorname{symb}\left(\widetilde{\rho}_{0}(n-1)\right), \operatorname{symb}(\hat{O})\right\rangle=\left\langle Q_{n} f, O\right\rangle \leq\left\|Q_{n} f\right\|\|O\|_{\infty}<\varepsilon
$$

Therefore, from Eq. (44) it follows that $\left(\widetilde{\rho}_{0}(n-1) \mid \hat{O}\right) \longrightarrow 0$.

\subsection{Quantum Ergodic Hierarchy Levels: Ergodic, Mixing and Exact}

Theorems 7 and 8 can be used simultaneously to determine ergodicity, mixing or exactness just by looking at the terms in the sums on the right hand side of Eq. (6) or Eq. (9). This observation holds also for the Quantum Spectral Decomposition Theorem (QSDT, Theorem 9) simply because this is a translation to the quantum language of its original version for dynamical systems. Therefore, we can apply theorem 8 and QSDT to obtain the following theorem in order to characterize the Quantum Ergodic Hierarchy levels (see [2] theorems 1,2 pags. 261, 263). 
Theorem 10. (Quantum Ergodic Hierarchy levels: Ergodic, Exact and Mixing) Let $S$ be a quantum system. Let $\hat{\rho} \in \mathcal{N}$ and let $\hat{O}$ be an observable. If $\hat{U}$ is a time evolution operator (i.e. representing a discretized time evolution in the sense of remark (I) of section 4) whose classical analogue $T$ (i.e. representing the time evolution of the analogue classical system in the sense of remark (II) of section 4) has a constrictive Markov operator P. Then

(I) $\hat{U}$ is ergodic $\Longleftrightarrow$ permutation $\{\alpha(1), \ldots, \alpha(r)\}$ of sequence $\{1, \ldots, r\}$ is cyclical (that is, for which there no is invariant subset).

(II) If $r=1$ in representation of $E q .(\underline{25}) \Longrightarrow \hat{U}$ is exact.

(III) If $\hat{U}$ is mixing (see [2] theorem 1 pag. 261) $\Longrightarrow r=1$ in representation of Eq. (25).

Again, as in theorem 8 we have $\hat{U}$ exact $\Longleftrightarrow \hat{U}$ mixing $\Longleftrightarrow r=1$.

Proof. It is enough to use Theorems 8 and 9 simultaneously.

In following subsections we examine the QEH levels established by Theorem 10 and its consequences in more detail. Since Theorem 10 does not distinguish between mixing and exact we will only discuss the mixing case (see remark 5.5.1 of [1]).

\subsubsection{A consequence of QSDT: Homogenization of the mixing level}

Consider a quantum system that is mixing. Then from Theorem 10 and Eq. (25) it follows that

$$
(\hat{\rho}(n) \mid \hat{O})=\left(\hat{\rho} \mid \hat{O}_{1}\right)\left(\hat{\rho}_{1} \mid \hat{O}\right)+\left(\widetilde{\rho}_{0}(n-1) \mid \hat{O}\right)
$$

where $\hat{\rho}_{1}$ is a pure state (in the classical limit) and $\hat{O}_{1}$ is an observable which does not depend on the observable $\hat{O}$. Further, since quantum system is mixing then it has a weak limit $\hat{\rho}_{*}$ such that $\lim _{n \rightarrow \infty}(\hat{\rho}(n) \mid \hat{O})=\left(\hat{\rho}_{*} \mid \hat{O}\right)$. From this limit and Eq. (45) we have

$$
\left(\hat{\rho}_{*} \mid \hat{O}\right)=\lim _{n \rightarrow \infty}\left(\hat{\rho} \mid \hat{O}_{1}\right)\left(\hat{\rho}_{1} \mid \hat{O}\right)+\lim _{n \rightarrow \infty}\left(\widetilde{\rho}_{0}(n-1) \mid \hat{O}\right)=\left(\hat{\rho} \mid \hat{O}_{1}\right)\left(\hat{\rho}_{1} \mid \hat{O}\right)
$$

since $\lim _{n \rightarrow \infty}\left(\widetilde{\rho}_{0}(n-1) \mid \hat{O}\right)=0$ (see (III) of Theorem 9$)$. Now, if we make $\hat{O}=\hat{1}$ in Eq. (46) given that $\left(\hat{\rho}_{*} \mid \hat{1}\right)=\operatorname{tr}\left(\hat{\rho}_{*}\right)=1$ and $\left(\hat{\rho}_{1} \mid \hat{1}\right)=\operatorname{tr}\left(\hat{\rho}_{1}\right)=1$ we obtain $\left(\hat{\rho} \mid \hat{O}_{1}\right)=1$ then $\left(\hat{\rho}_{*} \mid \hat{O}\right)=\left(\hat{\rho}_{1} \mid \hat{O}\right)$ for all $\hat{O}$ observable. Therefore, $\hat{\rho}_{1}=\hat{\rho}_{*}$. In other words, mixing level is physically responsible for the homogenization of $\hat{\rho}$ and its evolution towards the weak limit $\hat{\rho}_{1}$ which is a pure state. In this sense we can say that QSDT gives a physical interpretation of the mixing level.

\subsubsection{The ergodic level: Oscillation plus a term going to zero}

From Theorem 10 we can obtain a necessary and sufficient condition for ergodicity. A quantum system is ergodic if and only if permutation $\alpha$ of

$$
(\hat{\rho}(n) \mid \hat{O})=\sum_{i=1}^{r}\left(\hat{\rho} \mid \hat{O}_{\alpha^{-n}(i)}\right)\left(\hat{\rho}_{i} \mid \hat{O}\right)+\left(\widetilde{\rho}_{0}(n-1) \mid \hat{O}\right)
$$

is cyclical and $\left(\widetilde{\rho}_{0}(n-1) \mid \hat{O}\right) \rightarrow 0$. Since $\alpha$ is cyclical there is an integer $N>0$ such that $\alpha^{N}(i)=i$ and $\alpha^{-N}(i)=i$ for $i=1, \ldots, r$. That is, the inverse permutation $\alpha^{-1}$ operates on the indices $i=1, \ldots, r$ as 


$$
(1, \ldots, r) \longrightarrow^{\alpha^{-1}}(2,3, \ldots, r-2, r-1, r, 1) \longrightarrow^{\alpha^{-2}}(3,4, \ldots, r-1, r, 1,2) \ldots \longrightarrow^{\alpha^{-k}} \ldots \longrightarrow^{\alpha^{-N}}(1, \ldots, r)
$$

After $N$ successive steps we are back to the original cycle $(1, \ldots, r)$. This behavior indicates that sum of Eq. (47) will also return to its original value after $N$ successive time instants. Then sum of Eq. (47) is periodic with a period equal to $N$, i.e. with the same period as cycle $\alpha^{-1}$. More precisely, we have

$$
\begin{aligned}
& (\hat{\rho}(0) \mid \hat{O})=\left(\hat{\rho} \mid \hat{O}_{1}\right)\left(\hat{\rho}_{1} \mid \hat{O}\right)+\left(\hat{\rho} \mid \hat{O}_{2}\right)\left(\hat{\rho}_{2} \mid \hat{O}\right)+\ldots+\left(\hat{\rho} \mid \hat{O}_{r-1}\right)\left(\hat{\rho}_{r-1} \mid \hat{O}\right)+\left(\hat{\rho} \mid \hat{O}_{r}\right)\left(\hat{\rho}_{r} \mid \hat{O}\right)+\left(\widetilde{\rho}_{0}(-1) \mid \hat{O}\right) \\
& (\hat{\rho}(1) \mid \hat{O})=\left(\hat{\rho} \mid \hat{O}_{2}\right)\left(\hat{\rho}_{1} \mid \hat{O}\right)+\left(\hat{\rho} \mid \hat{O}_{3}\right)\left(\hat{\rho}_{2} \mid \hat{O}\right)+\ldots+\left(\hat{\rho} \mid \hat{O}_{r}\right)\left(\hat{\rho}_{r-1} \mid \hat{O}\right)+\left(\hat{\rho} \mid \hat{O}_{1}\right)\left(\hat{\rho}_{r} \mid \hat{O}\right)+\left(\widetilde{\rho}_{0}(0) \mid \hat{O}\right) \\
& (\hat{\rho}(2) \mid \hat{O})=\left(\hat{\rho} \mid \hat{O}_{3}\right)\left(\hat{\rho}_{1} \mid \hat{O}\right)+\left(\hat{\rho} \mid \hat{O}_{4}\right)\left(\hat{\rho}_{2} \mid \hat{O}\right)+\ldots+\left(\hat{\rho} \mid \hat{O}_{1}\right)\left(\hat{\rho}_{r-1} \mid \hat{O}\right)+\left(\hat{\rho} \mid \hat{O}_{2}\right)\left(\hat{\rho}_{r} \mid \hat{O}\right)+\left(\widetilde{\rho}_{0}(1) \mid \hat{O}\right) \\
& \cdot \\
& \cdot \\
& (\hat{\rho}(N) \mid \hat{O})=\left(\hat{\rho} \mid \hat{O}_{1}\right)\left(\hat{\rho}_{1} \mid \hat{O}\right)+\left(\hat{\rho} \mid \hat{O}_{2}\right)\left(\hat{\rho}_{2} \mid \hat{O}\right)+\ldots+\left(\hat{\rho} \mid \hat{O}_{r-1}\right)\left(\hat{\rho}_{r-1} \mid \hat{O}\right)+\left(\hat{\rho} \mid \hat{O}_{r}\right)\left(\hat{\rho}_{r} \mid \hat{O}\right)+\left(\widetilde{\rho}_{0}(N-1) \mid \hat{O}\right) \\
& (\hat{\rho}(N+1) \mid \hat{O})=\left(\hat{\rho} \mid \hat{O}_{2}\right)\left(\hat{\rho}_{1} \mid \hat{O}\right)+\left(\hat{\rho} \mid \hat{O}_{3}\right)\left(\hat{\rho}_{2} \mid \hat{O}\right)+\ldots+\left(\hat{\rho} \mid \hat{O}_{r}\right)\left(\hat{\rho}_{r-1} \mid \hat{O}\right)+\left(\hat{\rho} \mid \hat{O}_{1}\right)\left(\hat{\rho}_{r} \mid \hat{O}\right)+\left(\widetilde{\rho}_{0}(N) \mid \hat{O}\right)
\end{aligned}
$$

Then $\sum_{i=1}^{r}\left(\hat{\rho} \mid \hat{O}_{\alpha^{-(N+1)(i)}}\right)\left(\hat{\rho}_{i} \mid \hat{O}\right)=\sum_{i=1}^{r}\left(\hat{\rho} \mid \hat{O}_{\alpha^{-1}(i)}\right)\left(\hat{\rho}_{i} \mid \hat{O}\right)$. That is, if we call $F(n)=$

$$
\sum_{i=1}^{r}\left(\hat{\rho} \mid \hat{O}_{\alpha^{-n}(i)}\right)\left(\hat{\rho}_{i} \mid \hat{O}\right) \text { then }
$$

$$
F(N+1)=F(1)
$$

Thus sum $F(n)$ is periodic with a period equal to $N$. Moreover, permutation $\alpha$ is cyclical then $N=r$. Therefore, we see that in the ergodic case the mean values are composed by an oscillating part plus a term which tends to zero for large times ( $n$ goes to $\infty)$. From this remark we can obtain a sufficient and necessary condition for ergodicity. Let $S$ be a quantum system having a constrictive markovian Frobenius-Perron operator $P$ associated with the temporal evolution $T$ of its classical analogue. Then we have

$(\bullet) S$ is ergodic $\Longleftrightarrow$ for all discretized time evolution 1 (with discrete time steps at constants intervals) any mean value $(\hat{\rho}(n) \mid \hat{O})$ has only two terms, one of which is an oscillatory function of $n$ (with a period $N$ equals to the number of terms $r$ of the QSDT decomposition given by the Eq. (25)) and the other goes to zero for $n \rightarrow \infty$.

In next section the physical interpretation of condition $(\bullet)$ is studied which is the key to the characterization of the ergodic systems spectrum.

\section{QSDT and the quantum spectrum}

In addition to the characterization of mean values form of quantum ergodic hierarchy QSDT provides also a link between QEH levels and spectrum. In this section we extend this relationship

\footnotetext{
${ }^{16}$ Again, here we are considering that $\hat{U}(n)$ is the one given by the Hamiltonian or at most given by the Floquet operator. That is, $\hat{U}(n)=e^{-i \frac{\hat{H}}{\hbar} \alpha n}$ (Hamiltonian evolution) or $\hat{U}(n)=[\hat{U}(\tau)]^{n}$ (Floquet evolution, see Eq. 4.1.14 of [4]).
} 
to discrete case and continuous case, and when both types of spectrum are present. We point out the formalism we use was introduced by Antoniou et al in order to a give a rigorous mathematical description on the algebraic formalism of quantum mechanics [37].

Moreover, although condition $(\bullet)$ of previous section is a general result it is of great interest to examine the particular case $\hat{U}(n)=e^{-i \frac{\hat{H}}{\hbar} \alpha n}$ (i.e. the evolution operator is given by the Hamiltonian) where $\alpha$ is a real parameter which defines the discrete time steps. In this section we study what conditions for ergodicity and mixing can be obtained under this assumption.

\subsection{Discrete spectrum}

Let $\hat{\rho} \in \mathcal{N}$ be a state and let $\hat{O}$ be an observable. For simplicity, we assume the spectrum is finite and discrete. Also, we assume real eigenvalues. Let $E_{1}, E_{2}, \ldots, E_{N}$ be the energies of the system with their corresponding frequencies $\omega_{1}=\frac{E_{1}}{\hbar}, \omega_{2}=\frac{E_{2}}{\hbar}, \ldots, \omega_{N}=\frac{E_{N}}{\hbar}$. Let $|1\rangle,|2\rangle, \ldots,|N\rangle$ be their corresponding eigenvectors. We express any initial state $\hat{\rho}$ in the basis $\{|i\rangle\}_{i=1}^{N}$ as

$$
\hat{\rho}=\sum_{i=1}^{N} \rho_{i}|i\rangle\left\langle i\left|+\sum_{j \neq j^{\prime}}^{N} \rho_{j j^{\prime}}\right| j\right\rangle\left\langle j^{\prime}\right|
$$

Then considering $\hat{U}(n)=e^{-i \frac{\hat{H}}{\hbar} \alpha n}$ the mean value of $\hat{O}$ in $\hat{\rho}$ after $n$ time steps is

$$
(\hat{\rho}(n) \mid \hat{O})=\sum_{i=1}^{N} \rho_{i} O_{i}+\sum_{j \neq j^{\prime}}^{N} \rho_{j j^{\prime}}^{*} O_{j j^{\prime}} e^{-i\left(\omega_{j}-\omega_{j}^{\prime}\right) \alpha n}
$$

On the other hand, by QSDT we have

$$
(\hat{\rho}(n) \mid \hat{O})=\sum_{i=1}^{r} \lambda_{\alpha^{-n}(i)}\left(\hat{\rho}_{i} \mid \hat{O}\right)+\left(\widetilde{\rho}_{0}(n-1) \mid \hat{O}\right)
$$

Then, combining Eqns. (52) and (253) we obtain

$$
\sum_{i=1}^{N} \rho_{i} O_{i}+\sum_{j \neq j^{\prime}}^{N} \rho_{j j^{\prime}}^{*} O_{j j^{\prime}} e^{-i\left(\omega_{j}-\omega_{j}^{\prime}\right) \alpha n}=\sum_{i=1}^{r} \lambda_{\alpha^{-n}(i)}\left(\hat{\rho}_{i} \mid \hat{O}\right)+\left(\widetilde{\rho}_{0}(n-1) \mid \hat{O}\right)
$$

From Eq. (54) we can make the following considerations.

(A): If we suppose the system is ergodic then $\sum_{i=1}^{r} \lambda_{\alpha^{-n}(i)}\left(\hat{\rho}_{i} \mid \hat{O}\right)$ is a periodic function and because $\left(\widetilde{\rho}_{0}(n-1) \mid \hat{O}\right) \rightarrow 0$ for $n \rightarrow \infty$ it follows that $\sum_{i=1}^{r} \lambda_{\alpha^{-n}(i)}\left(\hat{\rho}_{i} \mid \hat{O}\right)+\left(\widetilde{\rho}_{0}(n-1) \mid \hat{O}\right)$ is a quasi-periodic function of $n \amalg$. The left hand side of Eq. (154) contains the term $\sum_{i=1}^{N} \rho_{i} O_{i}$ that is constant and therefore the sum $\sum_{j \neq j^{\prime}}^{N} \rho_{j j^{\prime}}^{*} O_{j j^{\prime}} e^{-i\left(\omega_{j}-\omega_{j}^{\prime}\right) \alpha n}$ is a quasi-periodic function of $n$. (B): Now suppose $\omega_{1}=\frac{E_{1}}{\hbar}, \omega_{2}=\frac{E_{2}}{\hbar}, \ldots, \omega_{N}=\frac{E_{N}}{\hbar}$ are rationally related. This means that there exists $k_{i j}, l_{i j} \in \mathbb{N}$ such that

$$
\frac{\omega_{i}}{\omega_{j}}=\frac{k_{i j}}{l_{i j}} \quad \forall i, j=1, \ldots, N
$$

\footnotetext{
${ }^{17}$ If we add a function $g(n)$ going to zero for $n \rightarrow \infty$ to a periodic function $f(n)$ then the sum $f(n)+g(n)$ is a quasi-periodic function.
} 
Now if we put $\omega_{0}=\min \left\{\omega_{1}, \ldots, \omega_{N}\right\}$ then we havet8

$$
\omega_{j}=\frac{n_{j}}{m_{j}} \omega_{0} \quad \forall j=1, \ldots, N \text { and } n_{j}, m_{j} \in \mathbb{N}
$$

On the other hand we know that each term $\rho_{i j}^{*} O_{i j} e^{-i\left(\omega_{i}-\omega_{j}\right) \alpha t}+C . C$ of non-diagonal part of $(\hat{\rho}(n) \mid \hat{O})$ (see Eq. (52) ) has a period $T_{i j}=\frac{2 \pi}{\alpha\left(\omega_{i}-\omega_{j}\right)}$. The number $T_{i j}$ is not necessarily an integer and it depends on the values of $\omega_{i}, \omega_{j}, \alpha$. Moreover, using Eq. (56) we can write $T_{i j}$ as

$$
T_{i j}=\frac{2 \pi m_{i} m_{j}}{\alpha\left(n_{i}-n_{j}\right) \omega_{0}} \quad \forall i, j=1, \ldots, N
$$

Now the trick is to choose a parameter $\alpha$ such that non-diagonal part of $(\hat{\rho}(n) \mid \hat{O})$ is periodic. Consider the lowest common multiple of products $m_{i} m_{j}$ and the greatest common divisor of all differences $n_{i}-n_{j}$ denoted by $L C M\left\{m_{i} m_{j}\right\}$ and $G C D\left\{n_{i} m_{j}-n_{j} m_{i}\right\}$ respectively. If we choose $\alpha=\frac{2 \pi}{G C D\left\{n_{i} m_{j}-n_{j} m_{i}\right\} \omega_{0}}$ then it is not difficult to see that $\sum_{i \neq j}^{N} \rho_{i j}^{*} O_{i j} e^{-i\left(\omega_{i}-\omega_{j}\right) \alpha n}$ is a periodic function of $n$. For this, it is enough to show that each term $\rho_{i j}^{*} O_{i j} e^{-i\left(\omega_{i}-\omega_{j}\right) \alpha n}+C . C$ takes the same value as in $n=0$ (i.e. $\rho_{i j}^{*} O_{i j}+C$.C) after $n$ steps where $n=L C M\left\{m_{i} m_{j}\right\}$. If $n=L C M\left\{m_{i} m_{j}\right\}$ and $\alpha=\frac{2 \pi}{G C D\left\{n_{i} m_{j}-n_{j} m_{i}\right\} \omega_{0}}$ we have

$$
\begin{aligned}
& \rho_{i j}^{*} O_{i j} e^{-i\left(\omega_{i}-\omega_{j}\right) \alpha n}+C . C=\rho_{i j}^{*} O_{i j} e^{-i\left(\frac{n_{i}}{m_{i}}-\frac{n_{j}}{m_{j}}\right) \omega_{0} \alpha n}+C . C=\rho_{i j}^{*} O_{i j} e^{-i\left(\frac{n_{i} m_{j}-n_{j} m_{i}}{m_{i} m_{j}}\right) \omega_{0} \alpha n}+C . C= \\
& \rho_{i j}^{*} O_{i j} e^{-i\left(\frac{n_{i} m_{j}-n_{j} m_{i}}{m_{i} m_{j}}\right) \omega_{0} \frac{2 \pi}{G C D\left\{n_{i} m_{j}-n_{j} m_{i}\right\} \omega_{0}} L C M\left\{m_{i} m_{j}\right\}}+C . C= \\
& \rho_{i j}^{*} O_{i j} e^{-i 2 \pi\left(\frac{n_{i} m_{j}-n_{j} m_{i}}{G C D\left\{n_{i} m_{j}-n_{j} m_{i}\right\}} \frac{L C M\left\{m_{i} m_{j}\right\}}{m_{i} m_{j}}\right)}+C . C
\end{aligned}
$$

Since $n_{i} m_{j}-n_{j} m_{i}$ is divisible by $G C D\left\{n_{i} m_{j}-n_{j} m_{i}\right\}$ and $L C M\left\{m_{i} m_{j}\right\}$ is divisible by $m_{i} m_{j}$ then there exists $K_{i j}, L_{i j} \in \mathbb{Z}$ such that

$$
\begin{aligned}
K_{i j} & =\frac{n_{i} m_{j}-n_{j} m_{i}}{G C D\left\{n_{i} m_{j}-n_{j} m_{i}\right\}} \\
L_{i j} & =\frac{L C M\left\{m_{i} m_{j}\right\}}{m_{i} m_{j}}
\end{aligned}
$$

Thus combining Eqns. (58) and (59) we have

$$
\rho_{i j}^{*} O_{i j} e^{-i\left(\omega_{i}-\omega_{j}\right) \alpha n}+C . C=\rho_{i j}^{*} O_{i j} e^{-i 2 \pi K_{i j} L_{i j}}+C . C=\rho_{i j}^{*} O_{i j}+C . C
$$

Therefore $\sum_{j \neq j^{\prime}}^{N} \rho_{j j^{\prime}}^{*} O_{j j^{\prime}} e^{-i\left(\omega_{j}-\omega_{j}^{\prime}\right) n}$ is a periodic function of $n$. Now if we assume the system is ergodic then $\sum_{i=1}^{r} \lambda_{\alpha^{-n}(i)}\left(\hat{\rho}_{i} \mid \hat{O}\right)$ is a periodic function of $n$ (see section 4.2.2.). Then we have

\footnotetext{
${ }^{18}$ From Eq. (55) it follows that $\frac{\omega_{i}}{\omega_{0}}=\frac{k_{i 0}}{l_{i 0}}$ for all $j=1, \ldots, N$. Now if make $n_{i}=k_{i 0}$ and $m_{i}=l_{i 0}$ then we obtain the Eq. (56).

${ }^{19}$ C.C denotes the complex conjugate.
} 


$$
\sum_{i=1}^{N} \rho_{i} O_{i}+\sum_{j \neq j^{\prime}}^{N} \rho_{j j^{\prime}}^{*} O_{j j^{\prime}} e^{-i\left(\omega_{j}-\omega_{j}^{\prime}\right) n}-\sum_{i=1}^{r} \lambda_{\alpha^{-n}(i)}\left(\hat{\rho}_{i} \mid \hat{O}\right)=\left(\widetilde{\rho}_{0}(n-1) \mid \hat{O}\right)
$$

is a non-trivial periodic function of $n$ going to zero for $n \longrightarrow \infty$. Contradiction. Then $S$ is not ergodic as we assumed.

Summing up, given a quantum system $S$ of finite discrete spectrum with a constrictive and markovian Frobenius-Perron operator $P$ associated with the temporal evolution of its classical analogue and considering $\hat{U}(n)=e^{-i \frac{\hat{H}}{\hbar} \alpha n}$, from the considerations (A) and (B) we obtain the following conditions for ergodicity.

$(\star) S$ is ergodic $\Longrightarrow$ quantum mean values $(\hat{\rho}(n) \mid \hat{O})$ are quasi-periodic functions of $n$ for all state $\hat{\rho}$ and observable $\hat{O}$.

$(\star \star)$ If frequencies $\omega_{1}=\frac{E_{1}}{\hbar}, \omega_{2}=\frac{E_{2}}{\hbar}, \ldots, \omega_{N}=\frac{E_{N}}{\hbar}$ are rationally related $\Longrightarrow S$ is not ergodic.

It is interesting to note that if we associate frequencies $\omega_{1}=\frac{E_{1}}{\hbar}, \omega_{2}=\frac{E_{2}}{\hbar}, \ldots, \omega_{N}=\frac{E_{N}}{\hbar}$ with angular velocities $\omega_{1}, \omega_{2}, \ldots, \omega_{N}$ of $N$ independent and autonomous oscillators then the condition $(\star \star)$ is the same that the one obtained for rotation on the torus (see pag. 190-193 of [1]). A possible explanation for this fact is as follows. QSDT allows to express mean values $(\hat{\rho}(n) \mid \hat{O})$ of a quantum system $S$ such that classical properties like ergodicity, mixing can be translated into quantum language in the same manner as the Spectral Decomposition Theorem (SDT), i.e. looking the number of terms and the periodicity of SDT (QSDT) decomposition (see Eqns. (9) and (25) ). Put in other words, as well as SDT only gives conditions for classical ergodicity (mixing) and does not distinguish between two ergodic (mixing) dynamical systems the same happens with QSDT. If we have two quantum systems $S_{1}, S_{2}$ of finite discrete (and real) spectrum QSDT does not distinguish whether any of them is an harmonic oscillator, a particle in a box, etc. But it only says that $S_{1}$ or $S_{2}$ are not ergodic if any of them satisfy that $\omega_{1}=\frac{E_{1}}{\hbar}, \omega_{2}=$ $\frac{E_{2}}{\hbar}, \ldots, \omega_{N}=\frac{E_{N}}{\hbar}$ are rationally related. Equivalently, condition $(\star \star)$ can be read as

$(\star \star \star) S$ is ergodic $\Longrightarrow$ frequencies $\omega_{1}=\frac{E_{1}}{\hbar}, \omega_{2}=\frac{E_{2}}{\hbar}, \ldots, \omega_{N}=\frac{E_{N}}{\hbar}$ are not rationally related.

In the next section we examine the case of discrete complex eigenvalues.

\subsubsection{Discrete complex eigenvalues}

Now we consider that eigenvalues are complex and finite. This is the case of quantum systems described by an effective non-hermitian Hamiltonian $H_{\text {eff }}$ that appears in atomic, molecular, nuclear physics and in chemical reactions. Let $\hat{\rho} \in \mathcal{N}$ be a sate and let $\hat{O}$ be an observable. As in previous section, we assume that any state $\hat{\rho}$ after $n$ successive steps is given by $\hat{U}(n) \hat{\rho} \hat{U}^{\dagger}(n)$ where $U(n)=e^{-i \frac{H_{e f f}}{\hbar} \alpha n}$ and $\alpha \in \mathbb{R}$ defines the time steps. Let $E_{1}=\omega_{1}+i \gamma_{1}, E_{2}=\omega_{2}+$ $i \gamma_{2}, \ldots, E_{N}=\omega_{N}+i \gamma_{N}$ be the complex eigenvalues of $H_{\text {eff }}$. Non-Hermiticity of $H_{\text {eff }}$ yields two set of eigenvectors called $\langle\widetilde{1}|,\langle\widetilde{2}|, \ldots,\langle\widetilde{N}|$ left eigenvectors and $|1\rangle,|2\rangle, \ldots,|N\rangle$ right eigenvectors (see Eq. (2) of [28])

$$
H_{e f f}|j\rangle=E_{j}|j\rangle \quad, \quad \widetilde{\langle j}\left|H_{e f f}=\widetilde{\langle j}\right| E_{j} \quad j=1, \ldots, N
$$


satisfying conditions

$$
\begin{aligned}
& \langle\widetilde{j} \mid k\rangle=\delta_{j k} \quad j, k=1, \ldots, N \quad(\text { bi-orthogonality }) \\
& \sum_{j=1}^{N}|j\rangle\langle\widetilde{j}|=\mathbb{I} \quad \text { (completeness) }
\end{aligned}
$$

where $E_{j}=\omega_{j}+i \gamma_{j}$ gives the energy $\omega_{j}$ and the resonance width $-\gamma_{j}>0$ of $j$ th resonance (see pag. 3 of [28]). In a more realistic case (see section 6) we can suppose that only first $K$ eigenvalues are real with $1 \leq K \leq N$, i.e. $\gamma_{j}=0$ for all $j=1, \ldots, K$. Again, we express $\hat{\rho}$ in the basis (co-basis) $\{|j\rangle\}_{j=1}^{N}\left(\{\mid\langle\widetilde{k}|\}_{k=1}^{N}\right)$

$$
\hat{\rho}=\sum_{i=1}^{N} \rho_{i}|i\rangle\left\langle\widetilde{i}\left|+\sum_{j \neq j^{\prime}}^{N} \rho_{j j^{\prime}}\right| j\right\rangle\left\langle\widetilde{j^{\prime}}\right|
$$

From Eq. (64) we have

$$
\begin{gathered}
\left.\hat{\rho}(n)=\hat{U}(n) \hat{\rho} \hat{U}^{\dagger}(n)=\hat{U}(n)\left(\sum_{i=1}^{N} \rho_{i}|i\rangle \widetilde{i}\left|+\sum_{j \neq j^{\prime}}^{N} \rho_{j j^{\prime}}\right| j\right\rangle\left\langle\widetilde{j^{\prime}}\right|\right) \hat{U}(n)^{\dagger}= \\
\left.\sum_{i=1}^{K} \rho_{i}|i\rangle \widetilde{i}\left|+\sum_{j \neq j^{\prime}}^{K} \rho_{j j^{\prime}}^{*} e^{-i \frac{\left(\omega_{j}-\omega_{j}^{\prime}\right)}{\hbar} \alpha n}\right| j\right\rangle\left\langle\widetilde{j^{\prime}}\left|+\sum_{j=K+1}^{N} \rho_{j} e^{2 \alpha n \frac{\gamma_{j}}{\hbar}}\right| j\right\rangle\langle\widetilde{j}|+ \\
\sum_{j \neq j^{\prime}, j} \rho_{\text {or } j^{\prime} \in\{K+1, \ldots, N\}}^{*} \rho^{*} e^{-i \frac{\left(\omega_{j}-\omega_{j}^{\prime}\right)}{\hbar} \alpha n} e^{\frac{\left(\gamma_{j}+\gamma_{j}^{\prime}\right)}{\hbar} \alpha n}|j\rangle\left\langle\widetilde{j^{\prime}}\right|
\end{gathered}
$$

Coefficients of last two sums of Eq. (65) contains probabilities of transitions to eigenstates with complex eigenvalues called "quasi-stationary states" whose resonance width $-\gamma_{j}$ is related to the lifetime $\Gamma_{j}$ by $-\gamma_{j} \sim \frac{\hbar}{\Gamma_{j}}$, see pag. 559 of [38]. Physically, $|K+1\rangle,|K+2\rangle, \ldots,|N\rangle$ represent states of an open quantum system in interaction with an environment where the exponentially decreasing coefficients of last two sums Eq. (65) can be interpreted as probabilities of finding a particle in one of the states $|K+1\rangle,|K+2\rangle, \ldots,|N\rangle$ "inside the system".

Next step is to show how quasi-stationary states can be interpreted in terms of QSDT. From Eq. (65) the mean value of an observable $\hat{O}$ after $n$ time steps reads

$$
\begin{gathered}
(\hat{\rho}(n) \mid \hat{O})=\sum_{i=1}^{K} \rho_{i} O_{i}+\sum_{j \neq j^{\prime}}^{K} \rho_{j j^{\prime}}^{*} O_{j j^{\prime}} e^{-i \frac{\left(\omega_{j}-\omega_{j}^{\prime}\right)}{\hbar} \alpha n}+\sum_{j=K+1}^{N} \rho_{j} O_{j} e^{2 \alpha n \frac{\gamma_{j}}{\hbar}}+ \\
\sum_{j \neq j^{\prime}, j \text { or } j^{\prime} \in\{K+1, \ldots, N\}} \rho_{j j^{\prime}}^{*} O_{j j^{\prime}} e^{-i \frac{\left(\omega_{j}-\omega_{j}^{\prime}\right)}{\hbar} \alpha n} e^{\frac{\left(\gamma_{j}+\gamma_{j}^{\prime}\right)}{\hbar} \alpha n}
\end{gathered}
$$

Therefore, by QSDT we have 


$$
\begin{aligned}
& \sum_{i=1}^{r} \lambda_{\alpha^{-n}(i)}\left(\hat{\rho}_{i} \mid \hat{O}\right)+\left(\widetilde{\rho}_{0}(n-1) \mid \hat{O}\right)=\sum_{i=1}^{K} \rho_{i} O_{i}+\sum_{j \neq j^{\prime}}^{K} \rho_{j j^{\prime}}^{*} O_{j j^{\prime}} e^{-i \frac{\left(\omega_{j}-\omega_{j}^{\prime}\right)}{\hbar} \alpha n}+ \\
& \sum_{j=K+1}^{N} \rho_{j} O_{j} e^{2 \alpha n \frac{\gamma_{j}}{\hbar}}+\sum_{j \neq j^{\prime}, j} \sum_{\text {or } j^{\prime} \in\{K+1, \ldots, N\}} \rho_{j j^{\prime}}^{*} O_{j j^{\prime}} e^{-i \frac{\left(\omega_{j}-\omega_{j}^{\prime}\right)}{\hbar} \alpha n} e^{\frac{\left(\gamma_{j}+\gamma_{j}^{\prime}\right)}{\hbar} \alpha n}
\end{aligned}
$$

Since $\left(\widetilde{\rho}_{0}(n-1) \mid \hat{O}\right) \rightarrow 0$ for $n \rightarrow \infty$ and last two sums on the right hand of Eq. (67) are the only terms that decay then we have

$$
\begin{aligned}
& \sum_{i=1}^{r} \lambda_{\alpha^{-n}(i)}\left(\hat{\rho}_{i} \mid \hat{O}\right)=\sum_{i=1}^{K} \rho_{i} O_{i}+\sum_{j \neq j^{\prime}}^{K} \rho_{j j^{\prime}}^{*} O_{j j^{\prime}} e^{-i \frac{\left(\omega_{j}-\omega_{j}^{\prime}\right)}{\hbar} \alpha n} \\
& \left(\widetilde{\rho}_{0}(n-1) \mid \hat{O}\right)=\sum_{j=K+1}^{N} \rho_{j} O_{j} e^{2 \alpha n \frac{\gamma_{j}}{\hbar}}+\sum_{j \neq j^{\prime}, j} \sum_{o r j^{\prime} \in\{K+1, \ldots, N\}} \rho_{j j^{\prime}}^{*} O_{j j^{\prime}} e^{-i \frac{\left(\omega_{j}-\omega_{j}^{\prime}\right)}{\hbar} \alpha n} e^{\frac{\left(\gamma_{j}+\gamma_{j}^{\prime}\right)}{\hbar} \alpha n}
\end{aligned}
$$

From Eq. (68) and Theorem 10 we conclude that the number of "quasi-stationary" states $|K+1\rangle,|K+2\rangle, \ldots,|N\rangle$ determines chaotic nature of the quantum system. For instance, if we have only one real eigenvalue $E_{1}$ then we have maximum number of quasi-stationary states $|2\rangle,|3\rangle, \ldots,|N\rangle$. In this case $K=1$ and $\sum_{i=1}^{r} \lambda_{\alpha^{-n}(i)}\left(\hat{\rho}_{i} \mid \hat{O}\right)$ is equal to $\rho_{1} O_{1}$. Then $r=1$, i.e. the system is mixing. When $K=2$ we have two real eigenvalues $E_{1}, E_{2}$ and $\sum_{i=1}^{r} \lambda_{\alpha^{-n}(i)}\left(\hat{\rho}_{i} \mid \hat{O}\right)$ is equal to $\rho_{1} O_{1}+\rho_{2} O_{2}+\rho_{12}^{*} O_{12} e^{-i \frac{\left(\omega_{1}-\omega_{2}\right)}{\hbar} \alpha n}+\rho_{21}^{*} O_{21} e^{-i \frac{\left(\omega_{2}-\omega_{1}\right)}{\hbar} \alpha n}$ that (by the arguments of the consideration (B) of the previous section) is an oscillatory function of $n$ if $\omega_{1}, \omega_{2}$ are rationally related and taking $\alpha=\frac{2 \pi}{G C D\left\{n_{1} m_{2}-n_{2} m_{1}\right\} \omega_{0}}$, i.e. $\omega_{1}=\frac{n_{1}}{m_{1}} \omega_{0}$ and $\omega_{2}=\frac{n_{2}}{m_{2}} \omega_{0}$. Then by condition $(\bullet)$ of section 4.2 .2 we have the system is ergodic.

We can see that in the finite spectrum case we have a substantial difference between having real eigenvalues and complex eigenvalues. In the case of real eigenvalues (see condition ( $\star \star)$ of section 5.1) we have seen that natural frequencies rationally related imply the system is not ergodic. While in the complex case if the real part of eigenvalues are rationally related then from the first line of Eq. (68) it follows the system is ergodid 20 . Moreover, in the complex case the decay term of QSDT, i.e. $\left(\widetilde{\rho}_{0}(n-1) \mid \hat{O}\right)$, is exponentially decreasing and provided by resonances widths $\gamma_{j}$ as we can see from the Eq. (68).

Summing up, we see that QSDT gives a characterization of open quantum systems of discrete complex eigenvalues where exponentially decreasing terms of mean values (the right hand on second line of Eq. (68) $)$ are associated with the decay term of QSDT, $\left(\widetilde{\rho}_{0}(n-1) \mid \hat{O}\right)$. All the analysis described in this section will be illustrated in some detail with examples in section 6 .

\subsection{Continuous spectrum}

Now we assume that spectrum is continuous being $E_{\omega}=\hbar \omega \in[0, \infty)$ the energies, $|\omega\rangle$ the generalized eigenvectors and $\omega=\frac{E_{\omega}}{\hbar}$ the natural frequencies. Let $\hat{\rho}$ be a state and let $\hat{O}$ be an

\footnotetext{
${ }^{20}$ I.e., using the same arguments of (B) of section 5.1.
} 
observable. In order to obtain an approach to equilibrium we restrict the space of observables and consider only van Hove Observables (see [39, 40, for a more detail discussion). There is no loss of generality in this restriction since observables not belonging to van Hove space are not experimentally accessible (see [41] for a complete argument). The components of a van Hove observable $\hat{O}_{R}$ are $O_{R}\left(\omega, \omega^{\prime}\right)=O(\omega) \delta\left(\omega-\omega^{\prime}\right)+O\left(\omega, \omega^{\prime}\right)$ where $O(\omega) \delta\left(\omega-\omega^{\prime}\right), O\left(\omega, \omega^{\prime}\right)$ are singular and regular $2 \square$ part of $\hat{O}_{R}$ respectively. Then we can expand $\hat{O}$ in the basis $\left\{|\omega\rangle\langle\omega|,| \omega\rangle\left\langle\omega^{\prime}\right|\right\}$ as

$$
\hat{O}=\int_{0}^{\infty} O(\omega)|\omega\rangle\left\langle\omega\left|d \omega+\int_{0}^{\infty} \int_{0}^{\infty} O\left(\omega, \omega^{\prime}\right)\right| \omega\right\rangle\left\langle\omega^{\prime}\right|
$$

Therefore, mean value of $\hat{O}$ in $\hat{\rho}$ after $n$ time steps i: $2 Z$

$$
(\hat{\rho}(n) \mid \hat{O})=\int_{0}^{\infty} \rho(\omega)^{*} O(\omega) d \omega+\int_{0}^{\infty} \int_{0}^{\infty} \rho\left(\omega, \omega^{\prime}\right)^{*} O\left(\omega, \omega^{\prime}\right) e^{-i\left(\omega-\omega^{\prime}\right) \alpha n} d \omega d \omega^{\prime}
$$

On the other hand, by QSDT we have

$$
\begin{aligned}
& (\hat{\rho}(n) \mid \hat{O})=\sum_{i=1}^{r}\left(\hat{\rho} \mid \hat{O}_{\alpha^{-n}(i)}\right)\left(\hat{\rho}_{i} \mid \hat{O}\right)+\left(\widetilde{\rho}_{0}(n-1) \mid \hat{O}\right)= \\
& =\int_{0}^{\infty} \rho(\omega)^{*} O(\omega) d \omega+\int_{0}^{\infty} \int_{0}^{\infty} \rho\left(\omega, \omega^{\prime}\right)^{*} O\left(\omega, \omega^{\prime}\right) e^{-i\left(\omega-\omega^{\prime}\right) \alpha n} d \omega d \omega^{\prime}
\end{aligned}
$$

If we assume that $\rho\left(\omega, \omega^{\prime}\right)^{*} O\left(\omega, \omega^{\prime}\right) \in L^{1}([0, \infty) \times[0, \infty))$ then by Riemann-Lebesgue Lemma we have

$$
\int_{0}^{\infty} \int_{0}^{\infty} \rho\left(\omega, \omega^{\prime}\right)^{*} O\left(\omega, \omega^{\prime}\right) e^{-i\left(\omega-\omega^{\prime}\right) \alpha n} d \omega d \omega^{\prime} \longrightarrow 0
$$

when $n \longrightarrow \infty$. That is, using van Hove observables and assuming that $\rho\left(\omega, \omega^{\prime}\right)^{*} O\left(\omega, \omega^{\prime}\right) \in$ $L^{1}([0, \infty) \times[0, \infty))$ then the system is mixing.

Moreover, from Eqns. (70) and (72) it follows that $(\hat{\rho}(n) \mid \hat{O}) \longrightarrow\left(\hat{\rho}_{*} \mid \hat{O}\right)=\int_{0}^{\infty} \rho(\omega)^{*} O(\omega) d \omega$ as $n \longrightarrow \infty$ with $\hat{\rho}_{*}=\int_{0}^{\infty} \rho(\omega)^{*}|\omega\rangle\langle\omega| d \omega$.

Therefore, $\hat{\rho}$ has weak limit $\hat{\rho}_{*}$. Also, if system is mixing then by Theorem 10 we have $r=1$ in the sum of (71). Then we have

$$
\begin{aligned}
& (\hat{\rho}(n) \mid \hat{O})=\left(\hat{\rho} \mid \hat{O}_{1}\right)\left(\hat{\rho}_{1} \mid \hat{O}\right)+\left(\widetilde{\rho}_{0}(n-1) \mid \hat{O}\right)= \\
& =\int_{0}^{\infty} \rho(\omega)^{*} O(\omega) d \omega+\int_{0}^{\infty} \int_{0}^{\infty} \rho\left(\omega, \omega^{\prime}\right)^{*} O\left(\omega, \omega^{\prime}\right) e^{-i\left(\omega-\omega^{\prime}\right) \alpha n} d \omega d \omega^{\prime}
\end{aligned}
$$

Now since $\left(\hat{\rho} \mid \hat{O}_{1}\right)\left(\hat{\rho}_{1} \mid \hat{O}\right)$ and $\int_{0}^{\infty} \rho(\omega)^{*} O(\omega) d \omega$ are constants and obtain

$\int_{0}^{\infty} \int_{0}^{\infty} \rho\left(\omega, \omega^{\prime}\right)^{*} O\left(\omega, \omega^{\prime}\right) e^{-i\left(\omega-\omega^{\prime}\right) \alpha n} d \omega d \omega^{\prime} \longrightarrow 0,\left(\widetilde{\rho}_{0}(n-1) \mid \hat{O}\right) \longrightarrow 0$ then by Eq. (73) we

$$
\begin{aligned}
& \left(\hat{\rho} \mid \hat{O}_{1}\right)\left(\hat{\rho}_{1} \mid \hat{O}\right)=\int_{0}^{\infty} \rho(\omega)^{*} O(\omega) d \omega \\
& \left(\widetilde{\rho}_{0}(n-1) \mid \hat{O}\right)=\int_{0}^{\infty} \int_{0}^{\infty} \rho\left(\omega, \omega^{\prime}\right)^{*} O\left(\omega, \omega^{\prime}\right) e^{-i\left(\omega-\omega^{\prime}\right) \alpha n} d \omega d \omega^{\prime}
\end{aligned}
$$

\footnotetext{
${ }^{21}$ By regular we mean, among other properties, that $f\left(\omega, \omega^{\prime}\right)^{*} O\left(\omega, \omega^{\prime}\right) \in L^{1}([0, \infty) \times[0, \infty))$ for all $f\left(\omega, \omega^{\prime}\right)$.

${ }^{22}$ Again, $\hat{\rho}(n)=\hat{U}(n) \hat{\rho} \hat{U}^{\dagger}(n)$ with $U(n)=e^{-i \frac{\hat{H}}{\hbar} \alpha n}$ and $\alpha \in \mathbb{R}$.
} 
From Eq. (74) we see the physical interpretation of term $\left(\widetilde{\rho}_{0}(n-1) \mid \hat{O}\right)$ : It is the manifestation of Riemann-Lebesgue Lemma in closed quantum systems with continuous spectrum [26] where observable space is the van Hove space. Therefore, the fact that mixing systems with continuous spectrum are those with only one term $\left(\hat{\rho} \mid \hat{O}_{1}\right)\left(\hat{\rho}_{1} \mid \hat{O}\right)$ in decomposition given by Eq. (25) is a consequence of QSDT where Riemann-Lebesgue Lemma is contained in term $\left(\widetilde{\rho}_{0}(n-1) \mid \hat{O}\right)$. From these arguments it follows the following property.

Summing up, given a quantum system $S$ of continuous spectrum with a constrictive markovian Frobenius-Perron operator $P$ associated with temporal evolution of its classical analogue and considering $U(n)=e^{-i \frac{\hat{H}}{\hbar} \alpha n}$ we have

(*) $S$ is mixing and the sum of QSDT decomposition (see Eq. (25)) only contains the term $\left(\hat{\rho} \mid \hat{O}_{1}\right)\left(\hat{\rho}_{1} \mid \hat{O}\right)$ which is the constant part of quantum mean value of Eq. (70), i.e. $\int_{0}^{\infty} \rho(\omega)^{*} O(\omega) d \omega$. The term $\left(\widetilde{\rho}_{0}(n-1) \mid \hat{O}\right)$ is the manifestation of Riemann-Lebesgue Lemma.

\subsection{The General Case: Discrete and Continuous Spectrum}

If both types of spectrum are present according to Eqns. (52) and (70) we have

$$
\begin{aligned}
& (\hat{\rho}(n) \mid \hat{O})=\sum_{i=1}^{N} \rho_{i} O_{i}+\sum_{j \neq j^{\prime}}^{N} \rho_{j j^{\prime}}^{*} O_{j j^{\prime}} e^{-i\left(\omega_{j}-\omega_{j}^{\prime}\right) \alpha n}+ \\
& \int_{0}^{\infty} \rho(\omega)^{*} O(\omega) d \omega+\int_{0}^{\infty} \int_{0}^{\infty} \rho\left(\omega, \omega^{\prime}\right)^{*} O\left(\omega, \omega^{\prime}\right) e^{-i\left(\omega-\omega^{\prime}\right) \alpha n} d \omega d \omega^{\prime}
\end{aligned}
$$

where first two sums and last two integrals represent the discrete and continuous contributions to spectrum respectively. By QSDT we have

$$
(\hat{\rho}(n) \mid \hat{O})=\sum_{i=1}^{r}\left(\hat{\rho} \mid \hat{O}_{\alpha^{-n}(i)}\right)\left(\hat{\rho}_{i} \mid \hat{O}\right)+\left(\widetilde{\rho}_{0}(n-1) \mid \hat{O}\right)
$$

From Eqns. (75) and (76) we obtain

$$
\begin{aligned}
& \sum_{i=1}^{N} \rho_{i} O_{i}+\sum_{j \neq j^{\prime}}^{N} \rho_{j j^{\prime}}^{*} O_{j j^{\prime}} e^{-i\left(\omega_{j}-\omega_{j}^{\prime}\right) \alpha n}+ \\
& \int_{0}^{\infty} \rho(\omega)^{*} O(\omega) d \omega+\int_{0}^{\infty} \int_{0}^{\infty} \rho\left(\omega, \omega^{\prime}\right)^{*} O\left(\omega, \omega^{\prime}\right) e^{-i\left(\omega-\omega^{\prime}\right) \alpha n} d \omega d \omega^{\prime}= \\
& \sum_{i=1}^{r}\left(\hat{\rho} \mid \hat{O}_{\alpha^{-n}(i)}\right)\left(\hat{\rho}_{i} \mid \hat{O}\right)+\left(\widetilde{\rho}_{0}(n-1) \mid \hat{O}\right)
\end{aligned}
$$

From Eq. (77) we can analyze the different cases. Let $S$ be a quantum system having both types of spectrum as in Eq. (75). Suppose $S$ has a constrictive markovian Frobenius-Perron operator $P$ associated with the temporal evolution of its classical analogue. One would be tempted to think that this case is simply the superposition of discrete and continuous cases each separately, but this is not so. 
We begin with the ergodic case. From Theorem 10 and section 4.2 .2 we know that $S$ is ergodic if and only if sum on right hand of Eq. (77) is periodic and we see the only term on left hand of Eq. (77) that can be periodic is $\sum_{j \neq j^{\prime}}^{N} \rho_{j j^{\prime}}^{*} O_{j j^{\prime}} e^{-i\left(\omega_{j}-\omega_{j}^{\prime}\right) \alpha n}$. Here we see a substantial difference from discrete case where we have $S$ is ergodic $\Longrightarrow \sum_{j \neq j^{\prime}}^{N} \rho_{j j^{\prime}}^{*} O_{j j^{\prime}} e^{-i\left(\omega_{j}-\omega_{j}^{\prime}\right) \alpha n}$ is quasi-periodic (see condition $(\star)$ of section 5.1). Furthermore, the term $\left(\widetilde{\rho}_{0}(n-1) \mid \hat{O}\right)$ which goes to zero should be associated with the only decay term on left hand of Eq. (77), i.e. the "Riemann-Lebesgue term" $\int_{0}^{\infty} \int_{0}^{\infty} \rho\left(\omega, \omega^{\prime}\right)^{*} O\left(\omega, \omega^{\prime}\right) e^{-i\left(\omega-\omega^{\prime}\right) \alpha n} d \omega d \omega^{\prime}$. Then we have

$$
\begin{aligned}
& \sum_{i=1}^{N} \rho_{i} O_{i}+\sum_{j \neq j^{\prime}}^{N} \rho_{j j^{\prime}}^{*} O_{j j^{\prime}} e^{-i\left(\omega_{j}-\omega_{j}^{\prime}\right) \alpha n}+\int_{0}^{\infty} \rho(\omega)^{*} O(\omega) d \omega=\sum_{i=1}^{r}\left(\hat{\rho} \mid \hat{O}_{\alpha^{-n}(i)}\right)\left(\hat{\rho}_{i} \mid \hat{O}\right) \\
& \int_{0}^{\infty} \int_{0}^{\infty} \rho\left(\omega, \omega^{\prime}\right)^{*} O\left(\omega, \omega^{\prime}\right) e^{-i\left(\omega-\omega^{\prime}\right) \alpha n} d \omega d \omega^{\prime}=\left(\widetilde{\rho}_{0}(n-1) \mid \hat{O}\right)
\end{aligned}
$$

Therefore we can see that in the ergodic case decay term is only provided by continuous part of spectrum as a consequence of QSDT. By contrast, in the mixing case this situation is very different due to non-degeneration condition of quantum chaos. Let us see why this is so.

In the mixing case we have that $S$ is mixing if and only $r=1$ in the sum on right hand of Eq. (77) and this happens if and only if double sum on left hand of Eq. (77) is constant. In this case there is no oscillatory term or quasiperiodic term and all frequencies $\omega_{j}$ are equal, e.g. $\omega_{j}=\omega_{0}$ for all $j=1, \ldots, N$. Then energy levels are degenerated. But if we recall that non-degeneration is a necessary condition of quantum chaos then this situation should not be physically admissible. Thus the only possibility that $\omega_{j}$ are non-degenerated without any oscillatory or quasiperiodic term is if double sum $\sum_{j \neq j^{\prime}}^{N} \rho_{j j^{\prime}}^{*} O_{j j^{\prime}} e^{-i\left(\omega_{j}-\omega_{j}^{\prime}\right) \alpha n}$ of Eq. (77) can be approximated by a double integral in order to apply Riemann-Lebesgue Lemma. In other words, discrete part must be quasicontinuous, i.e. adjacent energy levels are very close. More precisely, considering discrete part is supported in an interval $[a, b]$ such that $[a, b] \cap[0, \infty)=\emptyset$ with $N$ equispaced frequencies $\omega_{j}=a+\frac{(j-1)(b-a)}{N-1}$ and $j=1, \ldots, N$ then sums on left hand of Eq. (177) must be replaced by

$$
\begin{aligned}
& \sum_{i=1}^{N} \rho_{i} O_{i} \longrightarrow \sum_{j=1}^{N} \rho\left(\omega_{j}\right) O\left(\omega_{j}\right) \Delta \omega_{j} \quad \Delta \omega_{j}=\frac{b-a}{N-1}, \Delta \omega_{j^{\prime}}=\frac{b-a}{N-1} \\
& \sum_{j \neq j^{\prime}}^{N} \rho_{j j^{\prime}}^{*} O_{j j^{\prime}} e^{-i\left(\omega_{j}-\omega_{j}^{\prime}\right) \alpha n} \longrightarrow \sum_{j, j^{\prime}=1}^{N} \sum_{j \neq j^{\prime}}^{N} \rho\left(\omega_{j}, \omega_{j}^{\prime}\right)^{*} O\left(\omega_{j}, \omega_{j}^{\prime}\right) e^{-i\left(\omega_{j}-\omega_{j}^{\prime}\right) \alpha n} \Delta \omega_{j} \Delta \omega_{j^{\prime}}
\end{aligned}
$$

where $\Delta \omega_{j}=\frac{b-a}{N-1}$ is the length of segment $\left[\omega_{j}, \omega_{j+1}\right]$ and $\Delta \omega_{j} \Delta \omega_{j^{\prime}}=\left(\frac{b-a}{N-1}\right)^{2}$ is the volume of square $\left[\omega_{j}, \omega_{j+1}\right] \times\left[\omega_{j^{\prime}}, \omega_{j^{\prime}+1}\right]$. Now since discrete part is quasicontinuous then frequencies $\omega_{j}$ are very close and we have $\Delta \omega_{j}=\frac{b-a}{N-1} \ll 1$, i.e. we can take limit $N \rightarrow \infty$ in sums of (79). In such case we can approximate sums of Eq. (79) by integrals and we have the replacement:24

\footnotetext{
${ }^{23}$ Since $[0, \infty)$ is the continuous part of spectrum and we assume non-degeneration.

${ }^{24} \mathrm{It}$ is understood that integrals $\iint_{[a, b] \times[a, b]}$ and $\iint_{[a, b] \times[a, b] \backslash\{(\omega, \omega): \omega \in[a, b]\}}$ of Eq. (80) are equal since $\{(\omega, \omega)$ : $\omega \in[a, b]\}$ is a zero measure set of plane $\left\{\left(\omega, \omega^{\prime}\right)\right\}=\mathbb{R}_{\geq 0} \times \mathbb{R}_{\geq 0}$.
} 


$$
\begin{aligned}
& \omega_{j} \longrightarrow \omega, \quad \Delta \omega_{j} \longrightarrow d \omega \quad, \quad \Delta \omega_{j} \Delta \omega_{j^{\prime}} \longrightarrow d \omega d \omega^{\prime} \\
& \sum_{j=1}^{N} \rho\left(\omega_{j}\right) O\left(\omega_{j}\right) \Delta \omega_{j} \longrightarrow \int_{[a, b]} \rho(\omega) O(\omega) d \omega \\
& \sum_{j, j^{\prime}=1}^{N} \sum_{j \neq j^{\prime}}^{N} \rho\left(\omega_{j}, \omega_{j}^{\prime}\right)^{*} O\left(\omega_{j}, \omega_{j}^{\prime}\right) e^{-i\left(\omega_{j}-\omega_{j}^{\prime}\right) \alpha n} \Delta \omega_{j} \Delta \omega_{j^{\prime}} \longrightarrow \\
& \iint_{[a, b] \times[a, b] \backslash\{(\omega, \omega): \omega \in[a, b]\}} \rho\left(\omega, \omega^{\prime}\right)^{*} O\left(\omega, \omega^{\prime}\right) e^{-i\left(\omega-\omega^{\prime}\right) \alpha n} d \omega d \omega^{\prime}= \\
& \iint_{[a, b] \times[a, b]} \rho\left(\omega, \omega^{\prime}\right)^{*} O\left(\omega, \omega^{\prime}\right) e^{-i\left(\omega-\omega^{\prime}\right) \alpha n} d \omega d \omega^{\prime}
\end{aligned}
$$

Therefore, for the quasicontinuous case Eq. (77) becomes

$$
\begin{aligned}
& \int_{[a, b]} \rho(\omega) O(\omega) d \omega+\int_{0}^{\infty} \rho(\omega)^{*} O(\omega) d \omega=\left(\hat{\rho} \mid \hat{O}_{1}\right)\left(\hat{\rho}_{1} \mid \hat{O}\right) \\
& \iint_{[a, b] \times[a, b]} \rho\left(\omega, \omega^{\prime}\right)^{*} O\left(\omega, \omega^{\prime}\right) e^{-i\left(\omega-\omega^{\prime}\right) \alpha n} d \omega d \omega^{\prime}+ \\
& \int_{0}^{\infty} \int_{0}^{\infty} \rho\left(\omega, \omega^{\prime}\right)^{*} O\left(\omega, \omega^{\prime}\right) e^{-i\left(\omega-\omega^{\prime}\right) \alpha n} d \omega d \omega^{\prime}=\left(\widetilde{\rho}_{0}(n-1) \mid \hat{O}\right)
\end{aligned}
$$

That is,

$$
\begin{aligned}
& \int_{[a, b] \cup[0, \infty)} \rho(\omega) O(\omega) d \omega=\left(\hat{\rho} \mid \hat{O}_{1}\right)\left(\hat{\rho}_{1} \mid \hat{O}\right) \\
& \iint_{[a, b] \times[a, b] \cup[0, \infty) \times[0, \infty)} \rho\left(\omega, \omega^{\prime}\right)^{*} O\left(\omega, \omega^{\prime}\right) e^{-i\left(\omega-\omega^{\prime}\right) \alpha n} d \omega d \omega^{\prime}=\left(\widetilde{\rho}_{0}(n-1) \mid \hat{O}\right)
\end{aligned}
$$

Now if we apply Riemann-Lebesgue to double integral of Eq. (82) then this integral can be associated with term $\left(\widetilde{\rho}_{0}(n-1) \mid \hat{O}\right)$. Moreover, we can see how quasicontinuous part $\left\{\omega_{j}=\right.$ $\left.a+\frac{(j-1)(b-a)}{N-1}: j=1, \ldots, N\right\}$ joins with continuous part $\omega \in[0, \infty)$ in the limit $N \rightarrow \infty$ expressed in integrals $\int_{[a, b] \cup[0, \infty)}$ and $\iint_{[a, b] \times[a, b] \cup[0, \infty) \times[0, \infty)}$ of Eq. (82).

Summing up, given a quantum system $S$ having both types of spectrum, discrete and continuous, with a constrictive and markovian Frobenius-Perron operator $P$ associated with the temporal evolution of its classical analogue we have

- $S$ is ergodic $\Longrightarrow$ nondiagonal term of the discrete part of any quantum mean value (i.e. the second term of the right hand of Eq. (75) ) is a periodic function of $n$.

- $S$ is mixing $\Longleftrightarrow$ discrete part is quasicontinuous. 


\section{QSDT applications}

In this section we apply the QSDT to two examples 25 to illustrate its physical relevance: Microwave billiards and a phenomenological Gamow model. As we pointed out in section 4 and 5.1.1, both examples are open quantum systems that can be described by an effective nonHermitian Hamiltonian.

\subsection{Quantum ergodic and quantum mixing: Microwave billiards}

Microwave billiards are special examples of scattering systems 4, 5. Typically, to determine the spectrum of such systems antennas are used as scattering channels. An external coupling determines the resonances positions and widths, and the spectrum of microwave billiards are modified by the presence of the coupling antennas. We can start with an expression for the scattering matrix (see 4 pag. 221)

$$
\hat{S}=\hat{1}-2 i \hat{W}^{\dagger} \frac{\hat{1}}{E-\hat{H}_{0}+i \hat{W} \hat{W}^{\dagger}} \hat{W}
$$

where $\hat{1}$ is the identity matrix, $\hat{H}_{0}$ is the undisturbed Hamiltonian assumed to be a $N \times N$ truncated matrix and matrix elements $W_{n k}$ of $\hat{W}$ contain information on the coupling strengths of the $k$ th channel to the $n$th resonance. The poles of scattering matrix are eigenvalues of the effective Hamiltonian

$$
\hat{H}=\hat{H}_{0}-i \hat{W} \hat{W}^{\dagger}
$$

Information on widths and shifts induced by antennas is completely contained in the eigenvalues of effective hamiltonian $\hat{H}$. Effective Hamiltonians of this type have been extensively used in nuclear physics [42, 43, 44, 45, 46, 47]. We are only interested in the limiting case of large coupling strengths where $\hat{H}$ is dominated by the term $-i \hat{W} \hat{W}^{\dagger}$. The key is to select a basis where this term is diagonal and $\hat{H}_{0}$ is treated as a perturbation. If we have $K$ channels without loss of generality we may assume that the $K$ vectors $\hat{w}_{k}$ with components $W_{n k}$ are mutually orthogonal,

$$
\hat{w}_{k}^{\dagger} \hat{w}_{l}=\sum_{n} W_{n k}^{*} W_{n l}=\left|\hat{w}_{k}\right|^{2} \delta_{k l}
$$

Then we can take the orthogonal basis formed by the $K$ vectors $\hat{\nu}_{k}=\frac{\hat{w}_{k}}{\left|\hat{w}_{k}\right|}$ and the $N-K$ vectors $\hat{u}_{\alpha}$ where $\hat{H}_{0}$ is diagonal in subspace generated by vectors $\hat{u}_{\alpha}$ (see [4] pag. 221 and 222). Using first order perturbation theory the eigenvalues of $\hat{H}$ are

$$
\begin{array}{ll}
E_{l}=\hat{\nu}_{l}^{\dagger} \hat{H}_{0} \hat{\nu}_{l}-i\left|\hat{w}_{l}\right| & l \leq K \\
E_{l}=\hat{u}_{l}^{\dagger} \hat{H}_{0} \hat{u}_{l} & l>K
\end{array}
$$

Now we can consider the Hamiltonian

\footnotetext{
${ }^{25}$ We omit exact case since by QSDT (Theorem 10) we have $\hat{U}$ exact $\Longleftrightarrow \hat{U}$ mixing.
} 


$$
\hat{\mathcal{H}}=\sum_{l=1}^{K} E_{l}\left|\nu_{l}\right\rangle\left\langle\widetilde{\nu_{l}}\left|+\sum_{m=K+1}^{N} E_{m}\right| u_{m}\right\rangle\left\langle\widetilde{u_{m}}\right|
$$

which is a first order approximation of effective Hamiltonian $\hat{H}$. We have renamed $\hat{\nu}_{l}, \hat{u}_{m}$ as $\left|\nu_{l}\right\rangle,\left|u_{m}\right\rangle$ respectively, and Hamiltonian $\hat{\mathcal{H}}$ has been expressed in two sums to analyze different cases according to the number of channels $K$. Also, left vectors $\left\langle\widetilde{\nu_{l}}\right|,\left\langle\widetilde{u_{m}}\right|$ are those defined by Eqns. (62) and (63). We consider an initial state $\hat{\rho}$ given by

$$
\hat{\rho}=\sum_{l=1}^{K} \sum_{l^{\prime}=1}^{K} \rho_{l l^{\prime}}\left|\nu_{l}\right\rangle\left\langle\widetilde{\nu_{l^{\prime}}}\left|+\sum_{m=K+1}^{N} \sum_{m^{\prime}=K+1}^{N} \rho_{m m^{\prime}}\right| u_{m}\right\rangle\left\langle\widetilde{u_{m^{\prime}}}\right|+\left\{\sum_{\sigma=1}^{K} \sum_{\lambda=K+1}^{N} \rho_{\sigma \lambda}\left|\nu_{\sigma}\right\rangle\left\langle\widetilde{u_{\lambda}}\right|+\text { h.c. }\right\}
$$

where $\rho_{i j}=\langle i|\hat{\rho}| j\rangle$ for all $i, j=1, \ldots, N$ and first two sums of Eq. (88) are diagonal blocks corresponding to the subspaces spanned by $\left\{\left|\nu_{l}\right\rangle\right\}_{l=1}^{K}$ and $\left\{\left|u_{m}\right\rangle\right\}_{m=K+1}^{N}$ respectively. Third term of Eq. (88) contains nondiagonal elements of $\hat{\rho}$ which connect subspaces spanned by $\left\{\left|\nu_{l}\right\rangle\right\}_{l=1}^{K},\left\{\left|u_{m}\right\rangle\right\}_{m=K+1}^{N}$ and h.c. denotes the hermitian conjugate operation. We rename $\hat{\nu}_{l}^{\dagger} \hat{H}_{0} \hat{\nu}_{l}$ and $\hat{u}_{m}^{\dagger} \hat{H}_{0} \hat{u}_{m}$ as $\gamma_{l}$ and $\omega_{m}$ for all $l, m$. Again, operator $\hat{U}(n)$ is given by $\hat{U}(n)=e^{-i \frac{\hat{\mathcal{H}}}{\hbar} \alpha n}$ so $\hat{\rho}$ after $n$ steps is

$$
\begin{aligned}
& \hat{\rho}(n)=\hat{U}(n) \hat{\rho} \hat{U}(n)^{\dagger}=e^{-i \frac{\hat{\mathcal{H}}}{\hbar} \alpha n} \hat{\rho} e^{i \frac{\hat{\mathcal{H}}^{\dagger}}{\hbar} \alpha n}=\sum_{l=1}^{K} \sum_{l^{\prime}=1}^{K} \rho_{l l^{\prime}} e^{-\frac{\left(\left|\hat{w}_{l}\right|+\left|\hat{w}_{l^{\prime}}\right|\right) \alpha n}{\hbar}} e^{\frac{-i\left(\gamma_{l}-\gamma_{l^{\prime}}\right) \alpha n}{\hbar}}\left|\nu_{l}\right\rangle\left\langle\widetilde{\nu_{l^{\prime}}}\right| \\
& +\sum_{m=K+1}^{N} \sum_{m^{\prime}=K+1}^{N} \rho_{m m^{\prime}} e^{\frac{-i\left(\omega_{m}-\omega_{m^{\prime}}\right) \alpha n}{\hbar}}\left|u_{m}\right\rangle\left\langle\widetilde{u_{m^{\prime}}}\right|+\left\{\sum_{\sigma=1}^{K} \sum_{\lambda=K+1}^{N} \rho_{\sigma \lambda} e^{-\frac{\left|\hat{w}_{\sigma}\right| \alpha n}{\hbar}} e^{\frac{-i\left(\gamma_{\sigma}-\omega_{\lambda}\right) \alpha n}{\hbar}}\left|\nu_{\sigma}\right\rangle\left\langle\widetilde{u_{\lambda}}\right|+\text { h.c. }\right\}
\end{aligned}
$$

Then the mean value of an observable $\hat{O}$ in $\hat{\rho}$ after $n$ steps is

$$
\begin{aligned}
& (\hat{\rho}(n) \mid \hat{O})=\operatorname{tr}(\hat{\rho}(n) \hat{O})=\sum_{l=1}^{K} \sum_{l^{\prime}=1}^{K} \rho_{l l^{\prime}} O_{l l^{\prime}} e^{-\frac{\left(\left|\hat{w}_{l}\right|+\left|\hat{w}_{l^{\prime}}\right|\right) \alpha n}{\hbar}} e^{\frac{-i\left(\gamma_{l}-\gamma_{l^{\prime}}\right) \alpha n}{\hbar}} \\
& +\sum_{m=K+1}^{N} \sum_{m^{\prime}=K+1}^{N} \rho_{m m^{\prime}} O_{m m^{\prime}} e^{\frac{-i\left(\omega_{m}-\omega_{m^{\prime}}\right) \alpha n}{\hbar}}+\left\{\sum_{\sigma=1}^{K} \sum_{\lambda=K+1}^{N} \rho_{\sigma \lambda} O_{\sigma \lambda} e^{-\frac{\left|\hat{w}_{\sigma}\right| \alpha n}{\hbar}} e^{\frac{-i\left(\gamma_{\sigma}-\omega_{\lambda}\right) \alpha n}{\hbar}}+h . c .\right.
\end{aligned}
$$

where $O_{i j}=\langle i|\hat{O}| j\rangle$ for all $i, j=1, \ldots, N$. From Eq. (90) we see that first and third sums decay exponentially as $n \rightarrow \infty$ while second sum oscillates according to frequency differences $\omega_{m}-\omega_{m^{\prime}}$. This remark is crucial in order to analyze different cases according to number of channels $K$. By QSDT we have

$$
(\hat{\rho}(n) \mid \hat{O})=\sum_{i=1}^{r}\left(\hat{\rho} \mid \hat{O}_{\alpha^{-n}(i)}\right)\left(\hat{\rho}_{i} \mid \hat{O}\right)+\left(\widetilde{\rho}_{0}(n-1) \mid \hat{O}\right)
$$


Considering the previous remark and comparing Eqns. (90) and (91) we have

$$
\begin{aligned}
& \sum_{i=1}^{r}\left(\hat{\rho} \mid \hat{O}_{\alpha^{-n}(i)}\right)\left(\hat{\rho}_{i} \mid \hat{O}\right)=\sum_{m=K+1}^{N} \sum_{m^{\prime}=K+1}^{N} \rho_{m m^{\prime}} O_{m m^{\prime}} e^{\frac{-i\left(\omega_{m}-\omega_{m^{\prime}}\right) \alpha n}{\hbar}} \\
& \left(\widetilde{\rho}_{0}(n-1) \mid \hat{O}\right)=\sum_{l=1}^{K} \sum_{l^{\prime}=1}^{K} \rho_{l l^{\prime}} O_{l l^{\prime}} e^{-\frac{\left(\left|\hat{w}_{l}\right|+\left|\hat{w}_{l^{\prime}}\right|\right) \alpha n}{\hbar}} e^{\frac{-i\left(\gamma_{l}-\gamma_{l^{\prime}}\right) \alpha n}{\hbar}}+\left\{\sum_{\sigma=1}^{K} \sum_{\lambda=K+1}^{N} \rho_{\sigma \lambda} O_{\sigma \lambda} e^{-\frac{\left|\hat{w}_{\sigma}\right| \alpha n}{\hbar}} e^{\frac{-i\left(\gamma_{\sigma}-\omega_{\lambda}\right) \alpha n}{\hbar}}+\text { h.c. }\right\}
\end{aligned}
$$

First line of Eq. (92) shows the oscillatory part of $(\hat{\rho}(n) \mid \hat{O})$ while second line of Eq. (92) expresses the decay terms of $(\hat{\rho}(n) \mid \hat{O})$. This can be considered as a "global" QSDT characterization of first order Hamiltonian spectrum of microwave billiards.

Going into more detail, we consider a rectangle microwave billiard whose quantum mean values (at first order) are given by Eq. (92). In this case unperturbed Hamiltonian $\hat{H}_{0}$ has frequencies $\frac{\gamma_{l}}{\hbar}=\frac{\hat{\nu}_{l}^{\dagger} \hat{H}_{0} \hat{\nu}_{l}}{\hbar}, \frac{\omega_{m}}{\hbar}=\frac{\hat{\hat{u}}_{m}^{\dagger} \hat{H}_{0} \hat{u}_{m}}{\hbar}$ which can be considered rationally related 20 for all $l=1, \ldots, K ; m=K+1, \ldots, N$ then by arguments of consideration (B) of section 5.1 we have double sum of the first line of Eq. (92) is a periodic function of $n$. Now varying number of channels $K$ we can obtain different chaotic transitions from integrable regime to chaotic one.

We begin with $K=0$ that corresponds to integrable case $\hat{W}=0$. Then $\left|\hat{w}_{l}\right|=0$ for all $l=1, \ldots, K$ in Eq. (86) and quantum mean values have no terms going to zero, e.g. all terms of $(\hat{\rho}(n) \mid \hat{O})$ in Eq. (90) are oscillatory. Since there is no term that tends to zero then QSDT does not apply in this case.

Case $1 \leq K<N-1$. In this case $\hat{W} \neq 0$ and we have an exponential decay of $\left(\widetilde{\rho}_{0}(n-1) \mid \hat{O}\right)$ with characteristic times $\tau_{l l^{\prime}}=\frac{\hbar}{\left|\hat{w}_{l}\right|+\left|\hat{w}_{l^{\prime}}\right|}, \tau_{\sigma}=\frac{\hbar}{\left|\hat{w}_{\sigma}\right|}$ for all $l, l^{\prime}, \sigma=1, \ldots, K$. Since frequencies $\omega_{l}$ are rationally related for all $m=K+1, \ldots, N$ then right hand of first line of Eq. (92) is a periodic function of $n$. Therefore, from QSDT we have that microwave billiard is ergodic for $1 \leq K<N-1$ (see end of section 5.1). This case corresponds to pseudointegrable regime $(K=1)$ and chaotic regime $(K>1)$.

Case $K=N-1$. When $K=N-1$ we have $\left(\widetilde{\rho}_{0}(n-1) \mid \hat{O}\right)$ decays exponentially with characteristic times $\tau_{l l^{\prime}}=\frac{\hbar}{\left|\hat{w}_{l}\right|+\left|\hat{w}_{l^{\prime}}\right|}, \tau_{\sigma}=\frac{\hbar}{\left|\hat{w}_{\sigma}\right|}$ for all $l, l^{\prime}, \sigma=1, \ldots, N-1$ and right hand of the first line of Eq. (92) has only one term, $\rho_{N N} O_{N N}$. Then by QSDT it follows that $r=1$ and this means that microwave billiard is mixing. Therefore, microwave billiard is mixing for $K=N-1$. This case corresponds to a fully chaotic regime with maximum number of terms that decay exponentially.

The case $K=N$ is physically excluded because all quantum mean values $(\hat{\rho}(n) \mid \hat{O})$ can not tend to zero.

Therefore, application of QSDT to microwave billiards says that increasing of coupling channel number $K$ can be interpreted as chaotic transitions from integrable $(K=0)$ to ergodic $(1<K \leq N-1)$ and from ergodic to mixing $(K=N-1)$.

\footnotetext{
${ }^{26}$ This assumption is reasonable since dimensions of billiard can be adjusted such that frequencies are rationally related. Indeed, since only frequencies $\frac{\omega_{m}}{\hbar}$ are related with term $\sum_{i=1}^{r}\left(\hat{\rho} \mid \hat{O}_{\alpha^{-n}}(i)\right)\left(\hat{\rho}_{i} \mid \hat{O}\right)$ (see first line of Eq. (92)) then it is enough to consider that frequencies $\frac{\omega_{m}}{\hbar}$ are rationally related.
} 


\subsection{Quantum mixing case: A phenomenological Gamow model}

Phenomenological Gamow model type [6, 7] is perhaps one of simplest and more illustrative examples of decoherence and approach to equilibrium of a quantum system. Our system is a single quantum oscillator embed in a environment composed of a large bath of noninteracting quantum oscillators which can be considered a continuum. Degeneracies of this system render it convenient for application of Hamiltonian analytic extension (see [6, 48, 49, 50]) in order to obtain a non-Hermitian effective Hamiltonian 27 given by

$$
\hat{H}=\sum_{k=0}^{\infty} z_{k}|k\rangle\langle\widetilde{k}|
$$

where $z_{k}=k\left(\omega_{0}-i \gamma_{0}\right)$ are complex eigenvalues (except $\left.z_{0}=\omega_{0}\right), k=0,1,2, \ldots$ Natural frequency of single oscillator is $\omega_{0}$ and $\gamma_{0}$ is associated with relaxation time $t_{R}$ by $t_{R}=\frac{\hbar}{\gamma_{0}}$ (see [7] pag. 288 ). In other words, $\gamma_{0}$ is inversely proportional to decay rate of dumping of single oscillator. We consider an initial state $\hat{\rho}$ given by

$$
\hat{\rho}=\sum_{k=0}^{\infty} \sum_{m=0}^{\infty} \rho_{k m}|k\rangle\langle\widetilde{m}|
$$

where $\rho_{k m}=\langle k|\widehat{\rho}| m\rangle$ and $\rho_{k k} \geq 0, \rho_{m k}=\rho_{k m}^{*}$ with $k, m \in \mathbb{N}_{0}$. State $\hat{\rho}$ after $n$ time steps is given by

$$
\hat{\rho}(n)=\rho_{00}|0\rangle\left\langle 0\left|+\sum_{k=1}^{\infty} \rho_{k k} e^{-2 k \frac{\gamma_{0}}{\hbar} n}\right| k\right\rangle\left\langle k\left|+\sum_{k=0}^{\infty} \sum_{m=0, k \neq m}^{\infty} \rho_{k m} e^{-\frac{\gamma_{0}}{\hbar}(k+m) n}\right| k\right\rangle\langle m|
$$

Then mean value of an observable $\hat{O}$ in $\hat{\rho}$ after $n$ time steps is

$$
\begin{aligned}
& (\hat{\rho}(n) \mid \hat{O})=\operatorname{tr}(\hat{\rho}(n) \hat{O})=\sum_{k=0}^{\infty}\langle\widetilde{k}|\hat{\rho}(n) \hat{O}| k\rangle=\sum_{k=0}^{\infty}\{\hat{\rho}(n) \hat{O}\}_{k k}=\sum_{k=0}^{\infty} \sum_{m=0}^{\infty}\{\hat{\rho}(n)\}_{k m}\{\hat{O}\}_{m k}= \\
& =\rho_{00} O_{00}+\sum_{k=1}^{\infty} \rho_{k k} O_{k k} e^{-2 k \frac{\gamma_{0}}{\hbar} n}+\sum_{k=0}^{\infty} \sum_{m=0, k \neq m}^{\infty} \rho_{k m} O_{k m} e^{-\frac{\gamma_{0}}{\hbar}(k+m) n}
\end{aligned}
$$

where $\langle n|\hat{O}| m\rangle=O_{n m}$ with $k, m \in \mathbb{N}_{0}$ and parenthesis with subindexes $\{\hat{\rho}(n) \hat{O}\}_{k k},\{\hat{\rho}(n)\}_{k m},\{\hat{O}\}_{m k}$ are notations for corresponding matrix elements of $\hat{\rho}(n) \hat{O}, \hat{\rho}(n)$ and $\hat{O}$ respectively. On the other hand by, QSDT we have

$$
\begin{aligned}
& \sum_{i=1}^{r}\left(\hat{\rho}_{\psi} \mid \hat{O}_{\alpha^{-n}(i)}\right)\left(\hat{\rho}_{i} \mid \hat{O}\right)+\left(\widetilde{\rho}_{0}(n-1) \mid \hat{O}\right)= \\
& =\rho_{00} O_{00}+\sum_{k=1}^{\infty} \rho_{k k} O_{k k} e^{-2 k \frac{\gamma_{0}}{\hbar} n}+\sum_{k=0}^{\infty} \sum_{m=0, k \neq m}^{\infty} \rho_{k m} O_{k m} e^{-\frac{\gamma_{0}}{\hbar}(k+m) n}
\end{aligned}
$$

\footnotetext{
${ }^{27}$ In fact, in this non-Hermitian case we have $\hat{H}=\sum_{k=0}^{\infty} z_{k}|k\rangle\left\langle\widetilde{k}\left|\neq \sum_{m=0}^{\infty} z_{m}^{*}\right| m\right\rangle\langle\widetilde{m}|=\hat{H}^{\dagger}$ due presence of complex eigenvalues $z_{k}(k \neq 0)$ with nonzero imaginary parts.
} 
Since first term $\rho_{00} O_{00}$ on right hand of Eq. (97) is constant and remaining terms tends to zero when $n \rightarrow \infty$ (due presence of decreasing exponentials) then sum on left hand of Eq. (97) must consists of only one term, i.e. $r=1$. From this fact it follows that $\widetilde{\rho}_{0}(n-1)=\hat{\rho}(n)-\rho_{00}|0\rangle\langle 0|$ is associated with decay modes $z_{k}(k \neq 0)$. Therefore, from Theorem 10 (II, III) it follows that Gamow model is mixing. Moreover, we can obtain weak limit of state $\hat{\rho}$ (see [2] section 6.3 def. B). From Eq. (97) we have

$$
(\hat{\rho}(n) \mid \hat{O})=\sum_{i=1}^{r}\left(\hat{\rho}_{\psi} \mid \hat{O}_{\alpha^{-n}(i)}\right)\left(\hat{\rho}_{i} \mid \hat{O}\right)+\left(\widetilde{\rho}_{0}(n-1) \mid \hat{O}\right) \longrightarrow \rho_{00} O_{00}=\rho_{00}\left(\hat{\rho}_{|0\rangle\langle 0|} \mid \hat{O}\right) \quad \text { for } n \longrightarrow \infty
$$

where $\hat{\rho}_{|0\rangle\langle 0|}=|0\rangle\langle 0|$. That is, $\hat{\rho}_{w}=\rho_{00}|0\rangle\langle 0|$ is the weak limit of $\hat{\rho}[8$

\section{Conclusions}

Assuming that the classical limit of a quantum system has a constrictive Markovian FrobeniusPerron operator associated with its classical evolution $T$ we presented a quantum version of Spectral Decomposition Theorem of Dynamical Systems (Theorems 6 and 7) we called Quantum Spectral Decomposition Theorem (QSDT, Theorem 9). QSDT gives a representation of expectation values of all observable $\hat{O}$ characterizing ergodic level of QEH by presence of an oscillatory term (see Eq. (49)) and at the same time contains Riemann-Lebesgue Lemma for van Hove observables in the mixing case (see Eq. (74)). Moreover, in the mixing case QSDT provides a physical interpretation of "homogenization" (see Eqns. (45) and (46)) which is represented by a pure state $\hat{\rho}_{1}$ that is weak limit of $\hat{\rho}$.

Considering that quantum evolution is given by $\hat{U}(n)=e^{-i \frac{\hat{H}}{\hbar} \alpha n}$, i.e. a discretized evolution at constants intervals where the parameter $\alpha \in \mathbb{R}$ defines the time step, QSDT links QEH levels with spectrum. More precisely, when the spectrum is discrete we have that quasi-periodicity of quantum mean values is a necessary condition for ergodicity and linear dependence in the ring of integers of energy frequencies $\omega_{1}=\frac{E_{1}}{\hbar}, \omega_{2}=\frac{E_{2}}{\hbar}, \ldots, \omega_{N}=\frac{E_{N}}{\hbar}$ is a sufficient condition for non-ergodicity (see condition ( $\star \star$ ) of section 5.1).

For the complex discrete case QSDT gives us a characterization of quasi-stationary states (see Eq. (68) ) where exponentially decreasing terms of any mean value $(\hat{\rho}(n) \mid \hat{O})$ are associated with decay term $\left(\widetilde{\rho}_{0}(n-1) \mid \hat{O}\right)$. This can be considered as a QSDT characterization of open quantum systems described by an effective and non-Hermitian Hamiltonian.

For the continuous case we have that mean values of van Hove observables are composed by two terms, diagonal and non-diagonal, whose physical interpretation can be analyzed by means of QSDT decomposition. The terms $\int_{0}^{\infty} \rho(\omega)^{*} O(\omega) d \omega$ and $\int_{0}^{\infty} \int_{0}^{\infty} \rho\left(\omega, \omega^{\prime}\right)^{*} O\left(\omega, \omega^{\prime}\right) e^{-i \frac{\left(\omega-\omega^{\prime}\right)}{\hbar} n} d \omega d \omega^{\prime}$ can be identified with $\left(\hat{\rho} \mid \hat{O}_{1}\right)\left(\hat{\rho}_{1} \mid \hat{O}\right)$ and $\left(\widetilde{\rho}_{0}(n-1) \mid \hat{O}\right)$ respectively, see Eq. (74)). In other words, mixing case corresponds to continuous spectrum with a van Hove algebra. When both spectra are present QSDT give us two conditions (see section 5.3).

- Quantum system is ergodic then non-diagonal term of discrete part of spectrum is a periodic function of $n$, see Eq. (78).

\footnotetext{
${ }^{28}$ Non-normalization of $\hat{\rho}_{w}=\rho_{00}|0\rangle\langle 0|$ is a consequence of non-Hermiticity of Hamiltonian $\hat{H}$ given by Eq. (93). This is so because if $\hat{O}=\hat{I}$ from Eq. (96) we have $\operatorname{tr}(\rho(n))$ is decreasing and $\operatorname{tr}(\rho(n))=(\rho(n) \mid \hat{I}) \rightarrow \rho_{00}$ when $n \rightarrow \infty$. Therefore, $0<\rho_{00} \leq 1$ and $\rho_{00}=1 \Longleftrightarrow \rho_{k k}=0$ for all $k \neq 0$.
} 
- Quantum system is mixing if and only if discrete part of spectrum can be approximated by a quasicontinuous, see Eqns. (79) and (80).

As we pointed out in section 5.3, the general case is not the superposition of discrete and continuous cases simultaneously. In the ergodic case periodic term is provided by discrete part of spectrum while decay term is contributed by continuous part of spectrum. On the other hand, in the mixing case both continuous part and discrete part provide decay terms associated with $\left(\widetilde{\rho}_{0}(n-1) \mid \hat{O}\right)$ where discrete part is necessarily quasi-continuous. Roughly speaking, we can say that continuous part "forces" to discrete part to be periodic in the ergodic case and to be quasi-continuous in the mixing case.

In Section 6 we apply QSDT to two examples, microwave billiards and a phenomenological Gamow type model. In the first case QSDT allows us to characterize chaotic transitions, from integrable regime to chaotic one, of microwave billiards in terms of the number of channels $K$. When there is no channels $(K=0)$ mean values $(\widehat{\rho}(n) \mid \widehat{O})$ are oscillatory which corresponds to integrable regime. For a number of channels $K$ such that $1 \leq K<N-1$ [29 QSDT says that microwave billiard is ergodic which corresponds to pseudointegrable regime $(K=1)$ and chaotic regime $(K>1)$. This results due presence of terms that decay exponentially with a rate inversely proportional to the coupling strength $\left|\hat{w}_{\sigma}\right|$ for all $\sigma=1, \ldots, K$ (see Eq. (92)). This seems physically reasonable since a large coupling strength $\left|\hat{w}_{\sigma}\right| \gg 1$ implies a high scattering channel which corresponds to a small characteristic time $\tau_{\sigma}=\frac{\hbar}{\left|\hat{w}_{\sigma}\right|} \ll 1$, i.e. the exponential decay is fast. When $K=N-1$ QSDT says that microwave billiard is mixing which corresponds to fully chaotic regime where mean values $(\widehat{\rho}(n) \mid \widehat{O})$ contain a maximum number of terms that decay exponentially (see Eq. (92)). We summarize QSDT characterization of microwave billiards in the following table.

TABLE I: QSDT characterization of microwave billiard\$30

\begin{tabular}{||c|c|c||}
\hline \hline Number of channels $K$ & Quantum mean values $(\hat{\rho}(n) \mid \hat{O})$ & QEH level, regime \\
\hline$K=0$ & oscillatory & none, integrable \\
\hline $1 \leq K<N-1$ & exponential decay terms & ergodic, chaotic \\
\hline$K=N-1$ & $\begin{array}{c}\text { maximum number of } \\
\text { exponential terms } \\
\text { fully chaotic }\end{array}$ \\
\hline \hline
\end{tabular}

From Table I we see that increasing of number of channels implies an increasing of chaotic level. System starts with an integrable regime for $K=0$, enters to a chaotic ergodic regime when

\footnotetext{
${ }^{29} N$ is the dimension of truncated matrix of undisturbed Hamiltonian $\widehat{H}_{0}$.

${ }^{30}$ Again, we consider dimensions of billiard can be adjusted such that unperturbed Hamiltonian $\hat{H}_{0}$ has frequencies $\frac{\gamma_{l}}{\hbar}=\frac{\hat{\nu}_{l}^{\dagger} \hat{H}_{0} \hat{\nu}_{l}}{\hbar}, \frac{\omega_{m}}{\hbar}=\frac{\hat{u}_{m}^{\dagger} \hat{H}_{0} \hat{u}_{m}}{\hbar}$ which are rationally related.
} 
$1 \leq K<N-1$ and finally reaches a fully chaotic mixing regime for $K=N-1$, i.e. as number of antennas increases system becomes more chaotic. This can be considered as a QSDT characterization of microwave billiards in the limit of large coupling strengths.

In the case of Gamow model QSDT decomposition determines its mixing level, see Eq. (97). Moreover, given an initial state $\hat{\rho}$ its weak limit $\hat{\rho}_{w}=\rho_{00}|0\rangle\langle 0|$ can not be normalized due non-Hermiticity of effective Hamiltonian $\hat{H}$, see Eqns. (93) and (98).

We hope that all these features provided by QSDT can be be useful in shedding light on quantum chaos in future studies through more examples and theoretical essays.

\section{References}

[1] A. Lasota, M. Mackey, Probabilistic properties of deterministic systems, Cambridge Univ. Press, Cambridge, 1985.

[2] M. Castagnino, O. Lombardi , Phys. A, 388, 247-267, 2009.

[3] I. Gomez, M. Castagnino, Towards a definition of the Quantum Ergodic Hierarchy: Kolmogorov and Bernoulli systems, Physica A, 393, 112-131, 2014.

[4] H. Stockmann, Quantum Chaos - An Introduction, Cambridge Univ. Press, Cambridge, 1999.

[5] U. Stoffregen, J. Stein, H. Stockmann, M. Kus, F. Haake, Phys. Rev. Lett., 74, 2666, 1995.

[6] R. Laura, M. Castagnino, Phys. Rev. E, 57, 3948-3961, 1998.

[7] R. Omnès, The Interpretation of Quantum Mechanics, Princeton University, Princeton, 1994.

[8] G. Belot, J. Earman, Chaos out order: Quantum mechanics, the correspondence principle, and chaos, Stud. His. Philos. Mod. Phys. 28, 147-182, 1997.

[9] A. Lichtenberg, M. Lieberman, Regular and Chaotic Dynamics, Springer, New York, 1992.

[10] J. Guckenheimer, P. Holmes, Nonlinear oscillations, dynamical systems, and bifurcations of vector fields, Springer, Ithaca, 1985.

[11] J. Berkovitz, R. Frigg, F. Kronz, Stud. Hist. Phil. Mod. Phys., 37, 661-691, 2006.

[12] M. Gutzwiller, Chaos in Classical and Quantum Mechanics, Springer Verlag, New York, 1990.

[13] Y. Kuramoto, H. Araki Lecture Notes in Physics, International Symposium on Mathematical Problems in Theoretical Physics 39, Springer Verlag, New York, 1975.

[14] Y. Kuramoto, Chemical Oscillations, Waves, and Turbulence, Springer Verlag, New York, 1984.

[15] F. Haake, Quantum Signatures of Chaos, 2nd edition, Springer-Verlag, Heidelberg, 2001.

[16] G. Casati, B. Chirikov, Quantum Chaos: between order and disorder, Cambridge Univ. Press, Cambridge, 1995. 
[17] M. Tabor, Chaos and Integrability in Nonlinear Dynamics: An Introduction, Wiley, New York, 1988.

[18] M. Berry, Physica Scripta, 40, 335-336, 1989.

[19] J. Ford, G. Mantica, G. Ristow, The Arnol'd cat: Failure of the correspondence principle, Physica D, 50, 493-520, 1991.

[20] J. Ford, G. Mantica, Does quantum mechanics obey the correspondence principle? Is it complete?, Amer. J. Phys., 60, 1086-1098, 1992.

[21] H. Schuster, Deterministic Chaos, VCH, Weinheim, 1984.

[22] R. Batterman, Chaos, quantization and the correspondence principle, Synthese, 89, 189-227, 1991.

[23] S. Weinberg, Testing quantum mechanics, Ann. Phys., 194, 335-336, 1989.

[24] G. Ghirardi, A. Rimini, T. Weber, Unified dynamics for microscopic and macroscopic systems, Phys. Rev. D, 34, 470-491, 1986.

[25] I. Gomez, M. Castagnino, On the classical limit of quantum mechanics, fundamental graininess and chaos: Compatibility of chaos with the correspondence principle, Chaos, Solitons and Fractals, 68, 98-113, 2014.

[26] M. Castagnino, O. Lombardi, Self-induced decoherence and the classical limit of quantum mechanics, Philos. Sci., 72, 764-776, 2005.

[27] M. Sieber, Pramana-Journal Of Physics, 106, 121-167, 1984.

[28] U. Kuhl, O. Legrand, F. Mortessagne, Progress of Physics, 73, 543-551, 2009.

[29] I. Rotten, arXiv:0711.2926, 2007.

[30] N. Moiseyev, Non-Hermitian Quantum Mechanics, Cambridge Univ. Press, Cambridge, 2011.

[31] W. Slomczynski, K. Zyczkowski, J. Math. Phys., 35, 5674-5700, 1994.

[32] J. Paz, W. Zurek, Environment-induced decoherence and the transition from quantum to classical, Course 8 of Les Houches Lectures Session LXXII: Coherent Atomic Matter Waves, 533-614, Springer, Berlin, 2001.

[33] M. Hillery, M. O’Connell, R. Scully, E. Wigner, Phys. Rep., 106, 121-167, 1984.

[34] G. Dito, D. Sternheimer, arxiv math. QA/0201168, 2002.

[35] M. Gadella, Fortschr. Phys., 43, 229-264, 1995.

[36] W. Case, Am. J. Phys., 76, 937, 2008.

[37] I. Antoniou, Z. Sucha- necki, R. Laura, S. Tasaki, Intrinsic irreversibilty of quantum systems with diagonal singularity, Physica A, 241, 737-772, 1997. 
[38] L. Landau, E. Lifshitz, Quantum Mechanics: Non-Relativistic Theory, Pergamon Press, England, 1977.

[39] L. van Hove, Physica A, 20, 603, 1954.

[40] L. van Hove, Physica A, 25, 268, 1959.

[41] M. Castagnino, O. Lombardi, Stud. Hist. Phil. Mod. Phys., 35, 73, 2004

[42] C. Mahaux, H. Weidenmüller, Shell-Model Approach to Nuclear Reactions, North-Holland, Amsterdan, 1969.

[43] C. Lewenkopf, H. Weidenmüller, Ann. Phys., 212, 53, 1991.

[44] Y. Fyodorov, H. Sommers, J. Math. Phys., 38, 1918, 1997.

[45] E. Persson, T. Gorin, I. Rotter, Phys. Rev. E, 59, 3339, 1996.

[46] V. Sokoloff, V. Zelevinsky, Phys. Rev. Lett. B, 202, 10, 1988.

[47] H. Stockmann, P. Seba, J. Phys. A, 31, 3439, 1998.

[48] M. Gadella, G. Pronko, Fortschritte der Physik, 59, 795-859, 2011.

[49] M. Castagnino, S. Fortin, Modern Physics Letters A, 26, 2365-2373, 2011.

[50] G. Ordoñez, S. Kim, Phys. Rev. A, 70, 032702, 2004. 